AIAA AVIATION 2016

46th AIAA Fluid Dynamics Conference

June 13-17, 2016, Washington, DC

\title{
Tapping the Brake for Entry, Descent, and Landing
}

\author{
Peter A Gnoffo; Kyle Thompson ${ }^{\dagger}$ Ashley Korzun ${ }^{\ddagger}$ \\ NASA Langley Research Center, Hampton, VA 23681-2199
}

\begin{abstract}
A matrix of simulations of hypersonic flow over blunt entry vehicles with steady and pulsing retropropulsion jets is presented. Retropropulsion in the supersonic domain is primarily designed to reduce vehicle velocity directly with thrust. Retropropulsion in the hypersonic domain may enable significant pressure recovery through unsteady, oblique shocks while providing a buffer of reactant gases with relatively low total temperature. Improved pressure recovery, a function of Mach number squared and oblique shock angle, could potentially serve to increase aerodynamic drag in this domain. Pulsing jets are studied to include an additional degree of freedom to search for resonances in an already unsteady flow domain with an objective to maximize the time-averaged drag coefficient. In this paradigm, small jets with minimal footprints of the nozzle exit on the vehicle forebody may be capable of delivering the requisite perturbations to the flow. Simulations are executed assuming inviscid, symmetric flow of a perfect gas to enable a rapid assessment of the parameter space (nozzle geometry, plenum conditions, jet pulse frequency). The pulsedjet configuration produces moderately larger drag than the constant jet configuration but smaller drag than the jet-off case in this preliminary examination of a single design point. The fundamentals of a new algorithm for this challenging application with time dependent, interacting discontinuities using the feature detection capabilities of Walsh functions are introduced.
\end{abstract}

\section{Introduction}

Supersonic retro-propulsion is a proposed solution to landing high mass to the Martian surface. ${ }^{1}$ It addresses limitations of current systems to engage sufficient aerodynamic drag to land masses of order 10 metric tons or greater. Recent studies involving experimental ${ }^{2,3}$ and computational ${ }^{4}$ simulations have been engaged to better understand capabilities and limitations of this approach. These research efforts follow earlier studies that worked to define key parametric relationships that characterize both steady and unsteady interactions with the shock layer. ${ }^{5}$

Observations of these interactions reveal formation of oblique shocks and flows impinging on the vehicle surface. Total pressure losses are known to be high in stream tubes crossing strong shocks, with losses proportional to Mach number squared. It has been postulated that retro-firing jets can interact with the incoming flow to produce a larger fraction of the vehicle surface seeing flow processed by oblique shock(s) with the expectation that recovered pressures on the surface and the drag coefficient will be significantly higher. ${ }^{6,7}$ Moderately higher drag coefficients have been computed for these scenarios with steady firing jets; however, stream tubes processed by the oblique shocks tend to be turned by a buffer gas layer with total pressure substantially defined by normal shock processing.

The primary purpose of this work is to explore pulsed jet interactions with the incoming hypersonic flow to search for a resonance in which stream tubes processed by oblique shock(s) approach or exceed stagnation conditions on the outer extremities of an axisymmetric vehicle for significant fractions of the periodic interaction. The new idea here is to explore if the aerodynamic component of the interaction can be crafted to produce significantly larger retro-force than the thrust component. Pulsing provides an additional

\footnotetext{
* Senior Research Engineer, Aerothermodynamics Branch; AIAA Fellow

${ }^{\dagger}$ NASA Pathways Intern, Aerothermodynamics Branch; Student Member AIAA

${ }^{\ddagger}$ Atmospheric Flight and Entry Systems Branch; Member AIAA
} 
degree of freedom to sculpt the interaction for a desired outcome. If a reasonable fraction of the total pressure loss crossing strong shocks can be recovered, then doubling the drag coefficient in an average sense over the pulse duration may be possible.

Parameter space for this investigation (plenum pressure to total pressure ratio, nozzle area ratio, nozzle exit area to vehicle reference area ratio, and pulse frequency) will focus on axisymmetric, inviscid flow with a retro-firing nozzle positioned at the vehicle axis or multiple nozzles placed symmetrically around the axis. The primary simulation tool, FUN3D,$^{8,9}$ has been extensively tested for supersonic, retro-propulsion simulations. ${ }^{4}$ Here, we seek to identify how pulsed retro-firing interactions may be used to increase the drag coefficient. It is possible that optimal pulse frequencies are not sustainable in any practical rocket design. If that is the case, it may push future investigations to consider pulsed water jets or plasmas. Nevertheless, the ultimate goal is to design a simple, robust system with minimal footprint on the thermal protection system and with small propellant mass to provide more efficient braking at high Mach numbers.

The secondary purpose of this work is to explore the use of Walsh functions to provide effective feature detection in hypersonic flows with rapidly moving discontinuities. Retrofiring jets in a hypersonic flow are characterized by multiple shocks that rapidly fluctuate. The inviscid version of these simulations also include time dependent contact surface discontinuities. All of these moving discontinuities are captured in conventional, finite volume schemes, using upwind-based flux reconstruction algorithms. Best practices for capturing strong shocks call for aligning computational cells with the discontinuity. However, in a flow of this complexity, it is not possible to align all of the important flow discontinuities with the grid. Consequently, this complexity provides a challenging suite of tests for next-generation algorithms. Walsh functions can smoothly fit polynomials through discrete data (noisy or smooth) while automatically detecting discrete jumps in the data. This capability is being developed to provide some measure of tracking discontinuities in a computational domain. Progress to this end is described in the final section of this paper.

\section{Geometry and Grid}

All vehicle geometries considered herein are for a spherically capped cone with a circular shoulder with node radius $r_{\text {nose }}=0.25 \mathrm{~m}$, base radius $r_{\text {base }}=1.00 \mathrm{~m}$, and shoulder radius $r_{\text {shdr }}=0.100 \mathrm{~m}$. Most of the tested configurations have a cone half angle $\theta_{c}=70$ degrees. Two simulations for $\theta_{c}=55$ degrees and $\theta_{c}=45$ degrees are studied for effects of sonic line location on the jet interactions.

Three simple grid generation programs, appropriate for inviscid flows, are developed and utilized. All three programs employ simply-connected, multi-block, structured grids in Plot3 $\mathrm{D}^{10}$ format. (The hexagonal grids are converted to unstructured, AFLR $3^{11}$ format for use in FUN3D.) The first program is for axisymmetric flow with a single conical nozzle centered on the vehicle axis. The second program is also for axisymmetric flow, but with a single annular nozzle centered on the vehicle axis. In this case, the core of the nozzle is blocked so that the plume has a radial momentum component. The third program is for axisymmetric positioning of $N_{\text {noz }}$ nozzles on an axisymmetric vehicle. All of these programs are intended to provide a rapid prototyping of configurations for an initial assessment of jet interaction effectiveness. Desirable outcomes include a significant time-averaged increase in drag coefficient and/or a decrease in surface heating. Surface heating is not computed with an inviscid simulation, but contours of the total temperature field provide preliminary indications of relative heating trends. As the design space is explored and the mechanics of flow interaction are better understood, grid refinement studies and viscous simulations will be engaged at the most promising design points. The algebraic nature of the grid generation program is also expected to simplify future design iterations (at least for a subset of steady flows) driven by an adjoint solution with the objective of maximizing drag while minimizing propellant mass.

An overview of geometries and grids for the three configuration classes is shown in Fig. 1. Parameters defining configurations and grid resources are presented in Tables 1 and 2 for the single, conical nozzle, in Tables 3 and 4 for the single, annular nozzle, and in Tables 5 and 6 for the symmetric placement of five nozzles.

\section{A. Single, Axisymmetric, Conical Nozzle}

Three, simply connected, structured blocks defining the jet conical nozzle (block 1), the domain above the nozzle bounding the axis (block 2), and the domain covering the rest of the vehicle to the exit plane (block 3 ) are created. The geometric inputs enable definition of spherically capped cones with a circular shoulder and 


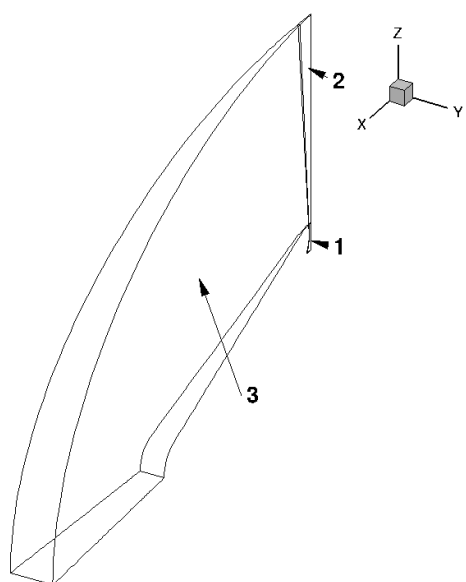

(a) View of block structure for conical nozzle

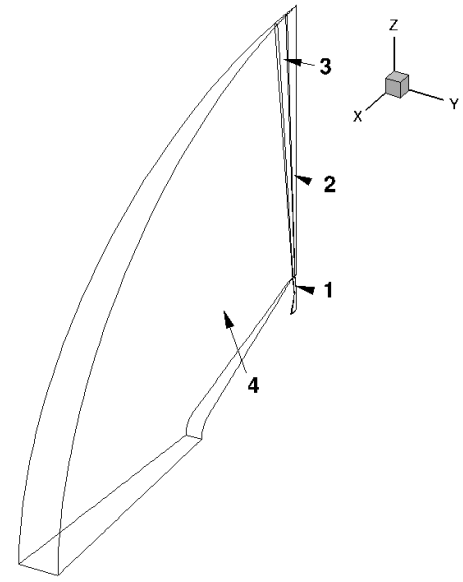

(c) View of block structure for annular nozzle

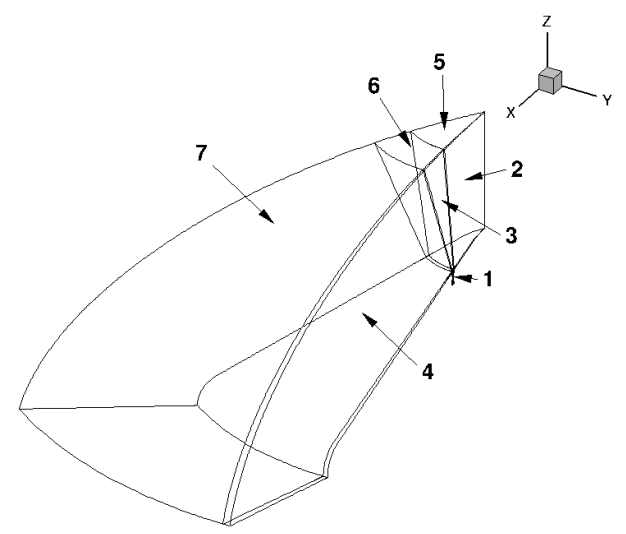

(e) View of block structure for symmetric, multiple nozzle

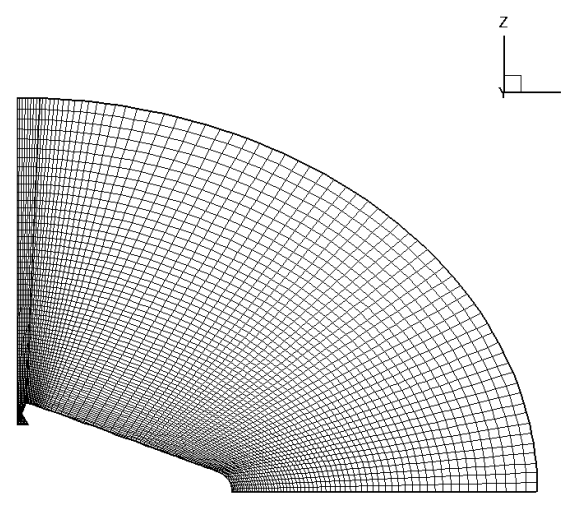

(b) Grid, Conical Nozzle

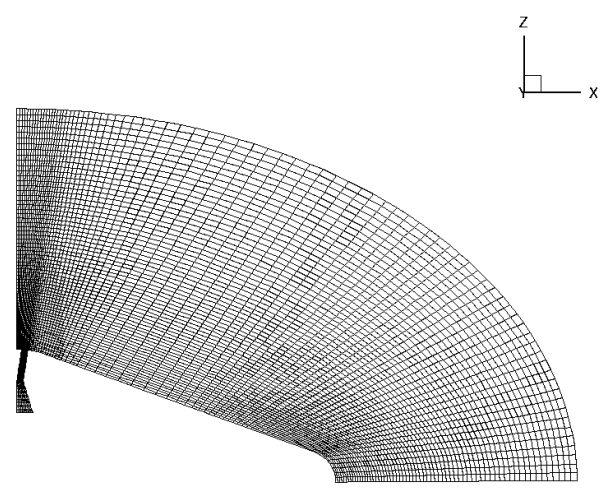

(d) Grid, Annular Nozzle

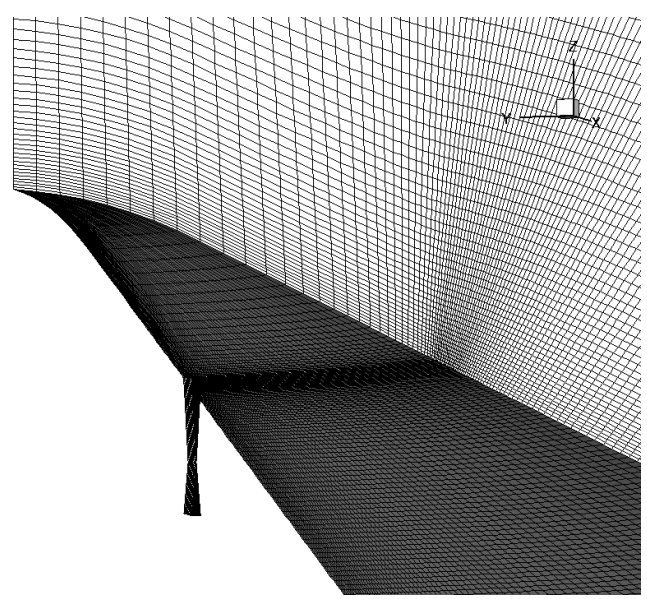

(f) Grid Detail, symmeric, multiple nozzles

Figure 1. Representative topologies and grids used in simulations.

a nozzle with an exit that is flush with the spherical nose. The geometry of a sample simulation is defined in Table 1. Grid parameters are defined in Table 2. The computational index $i$ runs from the axis of symmetry 
outward to the exit plane. The computational index $j$ defines the azimuthal angle. The computational index $k$ increases from the plenum interface outward to the inflow boundary. Equal spacing is used inside the nozzle and the mesh sizes are identical across the nozzle exit plane. The first cell off the vehicle has the same height as the adjacent cell at the nozzle exit. The parameters stretch, $N_{k, 2}$ and $N_{k, 3}$ in Table 2 may be used to control the position of the elliptical inflow boundary to accommodate large penetrations of the jet into the free stream. The resulting grid for configuration $\mathrm{C} 1$ is shown in Figs. 1a and 1b.

The nozzle geometry variation in Table 1 show a progressive decrease in the nozzle exit area. Configurations C2 - C4 employ a nozzle with a throat radius that is one half the radius (and one quarter the area) of configuration $\mathrm{C} 1$. The goal here is to constrict the jet such that a thinner plume that pierces the bow shock is ultimately defined.

Table 1. Vehicle Geometry Inputs, Conical Nozzle

\begin{tabular}{c|c|c|c|c|c} 
Parameter & Description & $\mathrm{C} 1$ & $\mathrm{C} 2$ & $\mathrm{C} 3$ & $\mathrm{C} 4$ \\
\hline$r_{\text {throat }}$ & nozzle throat radius, $\mathrm{m}$ & 0.020 & 0.010 & 0.010 & 0.010 \\
$r_{\text {plenum }}$ & nozzle radius at plenum interface, m & 0.050 & 0.025 & 0.025 & 0.025 \\
$r_{\text {exit }}$ & nozzle radius at exit, m & 0.040 & 0.020 & 0.015 & 0.012 \\
$l_{\text {conv }}$ & distance from plenum to throat, m & 0.050 & 0.050 & 0.050 & 0.050 \\
$l_{\text {div }}$ & distance from throat to exit, m & 0.050 & 0.050 & 0.050 & 0.050 \\
$\theta_{c}$ & cone half angle, deg & 70.00 & 70.00 & 70.00 & 70.00
\end{tabular}

Table 2. Grid Generation Inputs, Conical Nozzle

\begin{tabular}{c|c|r} 
Parameter & Description & C1-4 \\
\hline$N_{i, 1}$ & cells, $i$ direction, block 1, nozzle & 8 \\
$N_{k, 1}$ & cells, $k$ direction, block 1, nozzle & 16 \\
$N_{i, 2}$ & cells, $i$ direction, block 2, above nozzle exit & 8 \\
$N_{k, 2}$ & cells, $k$ direction, block 2, above nozzle exit & 64 \\
$N_{i, 3}$ & cells, $i$ direction, block 3, & 64 \\
$N_{k, 3}$ & cells, $k$ direction, block 3 & 64 \\
$N_{j}$ & cells, $j$ direction, all blocks & 1 \\
stretch & stretching factor, $k$ direction, blocks 2 and 3 & 1.02
\end{tabular}

\section{B. Single, Axisymmetric, Annular Nozzle}

Grid generation proceeds as described above for the conical nozzle except that now an inner and outer radial location for the annular nozzle exit are specified. See Table 3 for the annular nozzle configuration parameters and Table 4 for the associated grid allocations. Whereas the third block in the conical nozzle was situated over the nozzle exit it is now situated over the annular nozzle plug and a fourth block is placed over the annular exit. The stretching factor is reduced because previous simulations showed that the maximum displacement of the plume shock did not extend so far into the oncoming flow. The nozzle geometry for geometry A2 is twice as long as for A1, resulting in a smaller turning angle from the throat to the exit. The resulting grid for configuration A3 is shown in Figs. 1c and 1d.

\section{Multiple, Periodically Distributed Nozzles at a Fixed Radial Distance}

This program for multiple nozzles builds on the infrastructure developed above for the annular nozzle. It extends the logic to compute intersections of the nozzle centerline with any location on the vehicle surface. The radial location of the intersection of the nozzle centerline, $r_{n o z}$, and the angle of the nozzle centerline, $\theta_{\text {noz }}$ are specified. All cases described herein are for $\theta_{n o z}=0$. (A jet simulation with $\theta_{n o z}=45$ emanating from the shoulder at $r_{n o z}=0.96$ did not show any benefit for increasing drag.) The program extrudes (rotates) 
Table 3. Vehicle Geometry Inputs, Annular Nozzle

\begin{tabular}{c|c|c|c|c} 
Parameter & Description & A1 & A2 & A3 \\
\hline$r_{\text {throat }}$ & nozzle throat radius, m & 0.020 & 0.020 & 0.020 \\
$r_{\text {plenum }}$ & nozzle radius at plenum interface, m & 0.050 & 0.050 & 0.050 \\
$r_{\text {exit,in }}$ & inner nozzle radius at exit, m & 0.030 & 0.030 & 0.015 \\
$r_{\text {exit,out }}$ & outernozzle radius at exit, m & 0.050 & 0.050 & 0.035 \\
$l_{\text {conv }}$ & distance from plenum to throat, m & 0.050 & 0.100 & 0.100 \\
$l_{\text {div }}$ & distance from throat to exit, m & 0.050 & 0.100 & 0.100 \\
$\theta_{c}$ & cone half angle, deg & 70.00 & 70.00 & 70.00
\end{tabular}

Table 4. Grid Generation Inputs, Annular Nozzle

\begin{tabular}{c|c|r|r} 
Parameter & Description & A2-3 \\
\hline$N_{i, 1}$ & cells, $i$ direction, block 1, nozzle & 8 & 8 \\
$N_{k, 1}$ & cells, $k$ direction, block 1, nozzle & 16 & 32 \\
$N_{i, 2}$ & cells, $i$ direction, block 2, above nozzle plug & 8 & 8 \\
$N_{k, 2}$ & cells, $k$ direction, block 2, above nozzle plug & 64 & 64 \\
$N_{i, 3}$ & cells, $i$ direction, block 3, above nozzle exit & 8 & 8 \\
$N_{k, 3}$ & cells, $k$ direction, block 3, above nozzle exit & 64 & 64 \\
$N_{i, 4}$ & cells, $i$ direction, block 4, & 64 & 64 \\
$N_{k, 4}$ & cells, $k$ direction, block 4 & 64 & 64 \\
$N_{j}$ & cells, $j$ direction, all blocks & 1 & 1 \\
stretch & stretching factor, $k$ direction, blocks $2-4$ & 1.01 & 1.01
\end{tabular}

the resulting grid in the azimuthal direction to define a pie-shaped wedge with an included, azimuthal sweep angle, $\Phi_{S}$, equal to $180 / N_{n o z}$ degrees, where $N_{n o z}$ is the number of nozzles. A fifth block may by added beyond the shoulder to extend the body for cases where the nozzles are located at or near the shoulder of the vehicle. (See the discussion regarding extrapolation outflow boundary conditions in the next section.) The resulting grid for configuration N3f is shown in Figs. 1e and 1f. See Table 5 for the five-nozzle configuration parameters and Table 6 for the associated grid allocations.

The width of the nozzle is specified as a fraction, $f_{N}$, of the azimuthal sweep angle. Consequently, the nozzle included half angle, $\Phi_{N}$, is equal to $f_{N} \Phi_{S}$. The nozzle included half angle in Fig. 1f is $1 / 40$ of the azimuthal sweep angle. The resulting nozzle cross section is approximately rectangular using this algorithm. The number of simply connected blocks is now 7 (or 9 if the body extension is required) where the last 4 (or 5 , respectively) are the extruded versions of the blocks over the vehicle from $\Phi_{N}$ to $\Phi_{S}$. The extrusion algorithm computes a stretching factor based on the available number of cells in the azimuthal $(j$ coordinate direction) such that grid terminates exactly at $\Phi_{S}$.

The first three configurations, N1 - N3 have a nozzle location at the $20 \%$ radial distance with successively smaller throats. Configurations N4 and N5 have a nozzle location at the $60 \%$ radial distance. These two cases have the largest values of $\Phi_{N}$, the extruded nozzle half angle of all of the configurations. Configurations N6 and N7 place nozzles on lower angle cones for which the external flow over the nozzle is supersonic. It will be shown that these configurations tend to have a more steady plume shape as compared to jets firing in a subsonic domain.

\section{Boundary and Initial Conditions}

Inflow for all tests is at Mach 20. Complete details of the inflow conditions are defined in Table 7 . The choice of this single condition is motivated by the assumptions that (1) a strongly hypersonic flow has the greatest chance of generating favorable aerodynamic interactions because the pre-shock, total pressure is 
Table 5. Vehicle Geometry Inputs, Five Nozzles, $\Phi_{S}=36$ degrees

\begin{tabular}{c|c|c|c|c|c|c|c|c} 
Parameter & Description & $\mathrm{N} 1$ & $\mathrm{~N} 2$ & $\mathrm{~N} 3$ & $\mathrm{~N} 4$ & $\mathrm{~N} 5$ & $\mathrm{~N} 6$ & $\mathrm{~N} 7$ \\
\hline$r_{\text {throat }}$ & nozzle throat radius, $\mathrm{m}$ & 0.002 & 0.001 & 0.0002 & 0.005 & 0.020 & 0.002 & 0.020 \\
$r_{\text {plenum }}$ & nozzle radius at plenum, m & 0.005 & 0.005 & 0.005 & 0.030 & 0.040 & 0.005 & 0.040 \\
$r_{\text {exit }}$ & inner nozzle radius at exit, m & 0.005 & 0.005 & 0.005 & 0.010 & 0.040 & 0.005 & 0.040 \\
$r_{\text {noz }}$ & radial intersection of nozzle & 0.200 & 0.200 & 0.200 & 0.600 & 0.600 & 0.900 & 0.600 \\
& centerline with vehicle, $\mathrm{m}$ & & & & & & & \\
$\Phi_{N}$ & extruded nozzle half angle, deg & 0.9 & 0.9 & 0.9 & 6.0 & 1.2 & 0.9 & 1.2 \\
$l_{\text {conv }}$ & dist. from plenum to throat, m & 0.020 & 0.020 & 0.020 & 0.050 & 0.050 & 0.020 & 0.050 \\
$l_{\text {div }}$ & dist. from throat to exit, m & 0.030 & 0.030 & 0.030 & 0.050 & 0.050 & 0.030 & 0.060 \\
$\theta_{c}$ & cone half angle, deg & 70.00 & 70.00 & 70.00 & 70.00 & 70.00 & 55.00 & 45.00
\end{tabular}

Table 6. Grid Generation Inputs, Five Nozzles, $\Phi_{S}=36$ degrees

\begin{tabular}{c|c|r|r|r|r|r|r|r} 
Parameter & Description & N1, N2 & N1f, N3 & N3f & N4 & N5 & N6 & N7 \\
\hline$N_{i, 1}$ & cells, $i$ dir., block 1, nozzle & 4 & 8 & 16 & 8 & 8 & 4 & 8 \\
$N_{k, 1}$ & cells, $k$ dir., block 1, nozzle & 32 & 64 & 64 & 16 & 16 & 32 & 16 \\
$N_{i, 2,6}$ & cells, $i$ dir., block 2, inboard & 16 & 32 & 32 & 30 & 30 & 50 & 30 \\
$N_{k, 2,6}$ & cells, $k$ dir., block 2, inboard & 64 & 128 & 64 & 64 & 64 & 64 & 64 \\
$N_{i, 3,7}$ & cells, $i$ dir., block 3, above nozzle & 4 & 8 & 16 & 8 & 8 & 4 & 8 \\
$N_{k, 3,7}$ & cells, $k$ dir., block 3, above nozzle & 64 & 128 & 64 & 64 & 64 & 64 & 64 \\
$N_{i, 4,8}$ & cells, $i$ dir., block 4, outboard & 80 & 160 & 240 & 60 & 60 & 3 & 60 \\
$N_{k, 4,8}$ & cells, $k$ dir., block 4, outboard & 64 & 128 & 64 & 64 & 64 & 64 & 64 \\
$N_{i, 5,9}$ & cells, $i$ dir., block 5, extension, & 20 & 40 & NA & 32 & 32 & 20 & 32 \\
$N_{k, 5,9}$ & cells, $k$ dir., block 5, extension & 64 & 128 & NA & 64 & 64 & 64 & 64 \\
$N_{j, 1-5}$ & cells, $j$ dir. & 4 & 8 & 8 & 4 & 4 & 4 & 4 \\
$N_{j, 6-9}$ & cells, $j$ dir. & 32 & 64 & 64 & 8 & 32 & 32 & 32 \\
stretch & stretching factor, $k$ direction & 1.05 & 1.05 & 1.05 & 1.02 & 1.02 & 1.02 & 1.02
\end{tabular}

large; and (2) effects of gas chemistry will moderate but not obliterate any favorable aerodynamic interactions, if they exist. Note in Table 7 that the total pressure in the free stream is approximately four orders of magnitude larger than the total pressure behind the shock at Mach $20 .{ }^{12}$ Even if only a small fraction of that reservoir can be tapped to increase pressure loads on the forebody, then significant increase in the drag coefficient can be realized at a high Mach number. Tangency boundary conditions are applied on the vehicle and nozzle surfaces. The plenum interface cycles between a tangency boundary condition when the jet is off to a plenum boundary condition when the jet is on. The plenum boundary condition specifies the total pressure and temperature in the plenum. The outflow boundary is specified by first-order extrapolation. For tests where a jet is located near the outboard shoulder, the body is extended to prevent backflow through the wake of the jet plume. Side walls of the wedge-like domain are treated with appropriate symmetry and zero flow-through conditions. Initial conditions are defined by uniform flow at free stream values everywhere. (Note that the open end of the nozzles face the incoming flow so that this initialization does not lead to a near-vacuum transient that is often destabilizing.) Parameters defining jet plenum conditions are presented in Table 8 for the single, conical nozzle, in Table 9 for the single, annular nozzle, and in Table 10 for the symmetric placement of five nozzles. 
Table 7. Flow Simulation Parameters

\begin{tabular}{c|c|c} 
Parameter & Value & Description \\
\hline$V_{\infty}$ & 5686.24 & Velocity, $\mathrm{m} / \mathrm{s}$ \\
$\rho_{\infty}$ & 0.001 & Density, $\mathrm{kg} / \mathrm{m}^{3}$ \\
$T_{\infty}$ & 200.0 & Temperature, $\mathrm{K}$ \\
$M$ & 20 & Mach number, derived \\
$p_{t, \infty}$ & $2.7610^{8}$ & Total pressure in free stream, $\mathrm{N} / \mathrm{m}^{2}$, derived \\
$p_{t, 2}$ & $2.9710^{4}$ & Total pressure behind normal shock, $\mathrm{N} / \mathrm{m}^{2}$, derived \\
$T_{t, \infty}$ & 16200 & Total temperature in free stream, $\mathrm{K}$, derived \\
$t_{\text {flow }}$ & $1.75810^{-4}$ & $r_{\text {base }} / V_{\infty}, \mathrm{s}$ \\
$\Delta t$ & $0.001 t_{\text {flow }}$ & baseline simulation time step, $\mathrm{s}$
\end{tabular}

\section{Numerical Parameters}

In the simulations that follow, second-order spatial accuracy is specified using flux reconstruction defined by Roe's averaging ${ }^{13}$ and van Albada limiting. ${ }^{14}$ Eigenvalue limiter factors were set to 0.5 or 1.0. Lower values tended to enable carbuncle like flow features in the axisymmetric simulations for the jet off conditions - especially for cases in which a very fine grid resolving the core flow around the axis is required.

All simulations were run through the high energy, generic gas path of FUN3D, ${ }^{8,9}$ unless stated otherwise. A perfect-gas model for air is utilized for both incoming free stream and nozzle plenum conditions. FUN3D has the capability to run multiple species reacting gases in chemical and thermal non-equilibrium as well as different gas mixtures in the nozzle plenum; however, these options are not considered in this initial survey of unsteady flow interactions.

A time step equal to 0.001 flow times $\left(r_{\text {base }} /\left(1000 * V_{\infty}\right)=1.75710^{-7} \mathrm{~s}\right)$ is used throughout. Figure 2a compares the temporal variation of drag coefficient for configuration C3 in Table 1 at condition 6 in Table 8. A simulation with the baseline, dimensionless timestep, $\Delta t=0.001$ provides good agreement with a simulation using half that value for over five complete jet pulses occurring over 300 flow times.

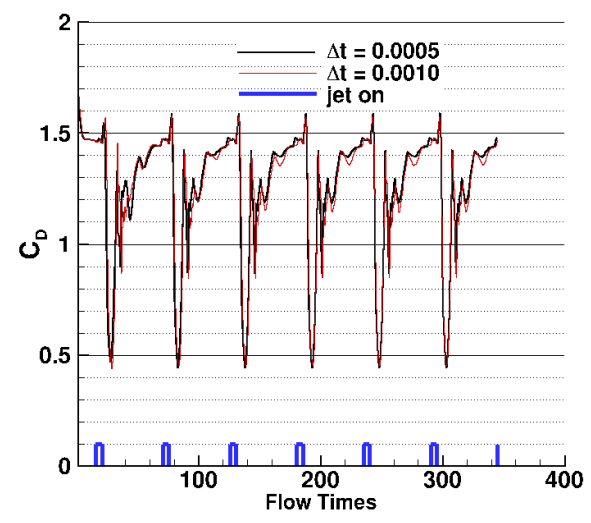

(a) Configuration C3 in Table 1 at condition 6 in Table 8. Time step refinement.

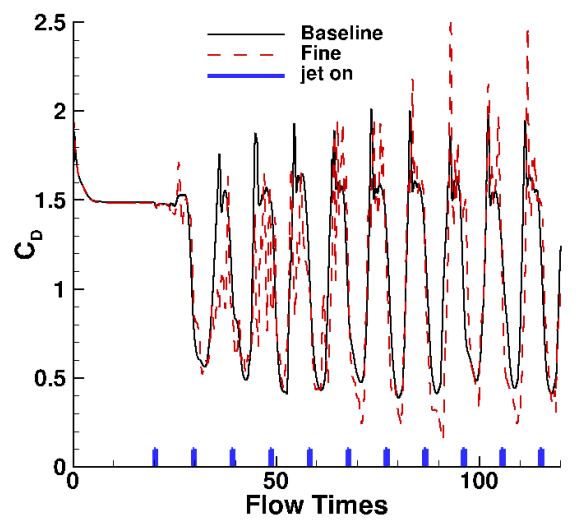

(b) Configuration $\mathrm{C} 4$ in Table 1 at condition 12 in Table 8. Time step and grid refinement.

Figure 2. Temporal and spatial grid convergence effects on the evolution of the drag coefficient for two conical nozzle cases. The jet pulses are indicated in blue at the bottom of the figure.

A second-order accurate temporal scheme is specified. Significant fluctuations of the bow shock are expected to compromise second-order temporal accuracy. Four sub-iterations are employed prior to advancing to the next time step. One typically sees one to two orders of magnitude decrease in the L2 norm over these 
four sub-iterations. As an example, the simulation for configuration N3f (Table 5) at condition number 1 in Table 10 indicated a mean flow residual that equaled $3.3810^{-7}$ after the first sub-iteration and dropped to $1.6310^{-8}$ after the fourth sub-iteration. In this same case, very little subsequent drop in the mean flow residual is observed if eight sub-iterations are engaged.

Retrofiring jets in a hypersonic flow are characterized by multiple shocks that rapidly fluctuate. The inviscid simulations also include time dependent contact surface discontinuities. Temporal and spatial grid refinement studies are the only tool available to characterize the accuracy of the simulations. To that end, a simulation for conical nozzle configuration C4 in Table 1 at condition 12 in Table 8 is implemented using both the baseline numerical parameters and a corresponding fine grid version. Both the spatial and temporal grid are refined by a factor of 2, the stretching parameter is reduced to 1.01, and the time step is halved to 0.0005 in the simulation labeled "Fine" in Fig. 2b. It is noted that the fine grid results in red appear to resolve more structure in the temporal evolution of the drag coefficient. Peaks and valleys are somewhat sharper and more structure is evident in the fine grid results where a relatively flat plateau is observed in the baseline results. Nevertheless, the overall character of the interactions are similar.

Table 8. Conical Nozzle Test Cases

\begin{tabular}{|c|c|c|c|c|c|}
\hline Geometry & Condition & $\begin{array}{c}p_{t, \text { plenum }} \\
\text { Plenum Total } \\
\text { Pressure, } \mathrm{N} / \mathrm{m}^{2}\end{array}$ & $\begin{array}{c}T_{t, \text { plenum }} \\
\text { Plenum Total } \\
\text { Temperature, } \mathrm{K}\end{array}$ & $\begin{array}{c}t_{\text {on }} / t_{\text {flow }} \\
\text { Pulse Duration }\end{array}$ & $\begin{array}{c}t_{\text {off }} / t_{\text {flow }} \\
\text { Time Between Pulses }\end{array}$ \\
\hline $\mathrm{C} 1$ & 1 & $5.0010^{4}$ & 1000 & on & $\mathrm{NA}$ \\
\hline $\mathrm{C} 1$ & 2 & $5.0010^{4}$ & 1000 & 2 & 2 \\
\hline $\mathrm{C} 1$ & 3 & $5.0010^{4}$ & 1000 & 10 & 10 \\
\hline $\mathrm{C} 2$ & 1 & $5.0010^{4}$ & 1000 & on & NA \\
\hline $\mathrm{C} 2$ & 2 & $1.0010^{5}$ & 1000 & on & NA \\
\hline $\mathrm{C} 2$ & 3 & $1.0010^{5}$ & 2000 & on & NA \\
\hline $\mathrm{C} 2$ & 4 & $1.0010^{5}$ & 4000 & on & NA \\
\hline C3 & 1 & $1.0010^{5}$ & 1000 & on & NA \\
\hline C3 & 2 & $2.0010^{5}$ & 1000 & 4 & 4 \\
\hline C3 & 3 & $2.0010^{5}$ & 1000 & 5 & 5 \\
\hline C3 & 4 & $2.0010^{5}$ & 1000 & 9 & 9 \\
\hline C3 & 5 & $2.0010^{5}$ & 1000 & 10 & 10 \\
\hline C3 & 6 & $2.0010^{5}$ & 1000 & 5 & 50 \\
\hline $\mathrm{C} 4$ & 1 & $2.0010^{5}$ & 1000 & 0.5 & 5 \\
\hline $\mathrm{C} 4$ & 2 & $2.0010^{5}$ & 1000 & 1 & 5 \\
\hline $\mathrm{C} 4$ & 3 & $2.0010^{5}$ & 1000 & 5 & 5 \\
\hline $\mathrm{C} 4$ & 4 & $3.0010^{5}$ & 1000 & 0.5 & 5 \\
\hline $\mathrm{C} 4$ & 5 & $4.0010^{5}$ & 1000 & 0.5 & 5 \\
\hline $\mathrm{C} 4$ & 6 & $5.0010^{5}$ & 1000 & 0.5 & 5 \\
\hline $\mathrm{C} 4$ & 7 & $6.0010^{5}$ & 1000 & 0.5 & 5 \\
\hline $\mathrm{C} 4$ & 8 & $7.0010^{5}$ & 1000 & 0.5 & 5 \\
\hline $\mathrm{C} 4$ & 9 & $7.0010^{5}$ & 1000 & 0.5 & 6 \\
\hline $\mathrm{C} 4$ & 10 & $7.0010^{5}$ & 1000 & 0.5 & 7 \\
\hline $\mathrm{C} 4$ & 11 & $7.0010^{5}$ & 1000 & 0.5 & 8 \\
\hline $\mathrm{C} 4$ & 12 & $7.0010^{5}$ & 1000 & 0.5 & 9 \\
\hline $\mathrm{C} 4$ & 13 & $7.0010^{5}$ & 1000 & 0.5 & 9.5 \\
\hline $\mathrm{C} 4$ & 14 & $7.0010^{5}$ & 1000 & 0.5 & 10 \\
\hline $\mathrm{C} 4$ & 15 & $7.0010^{5}$ & 1000 & 0.5 & 11 \\
\hline $\mathrm{C} 4$ & 16 & $7.0010^{5}$ & 1000 & 1 & 9 \\
\hline
\end{tabular}




\section{Retro-Propulsion Simulations}

Setting up an inviscid, axisymmetric, jet interaction problem and running 30,000 time steps can be completed in a half-day's work on an iMac desktop running a single processor with FUN3D. Most cases require more than 30,000 time steps to allow any periodic interactions to clearly repeat themselves. An implicit assumption in the following simulations is that if a resonant condition is identified that maximizes a time averaged drag coefficient, then that same resonance likely exists for more realistic gas models, albeit at somewhat different nozzle and plenum parameters. Conversely, if no such resonant condition for the simple gas model can be identified, then it is not likely to exist for a more realistic gas model. Note that jet plume density can be modified through changes to the plenum total temperature.

\section{A. Conical Nozzle}

The plenum conditions for all of the conical nozzle configurations are defined in Table 8 . In the pulsed jet scenario, the jet-on and jet-off durations for configuration $\mathrm{C} 1$ are identical in Table 8. The pulse duration is initially set to $t_{\text {on }}=2 t_{\text {flow }}$. If the pulse duration time is much longer than the time for the jet plume to reach equilibrium (steady or quasi-steady), then pulsing the jet is likely to be equivalent to the quasisteady solution for the jet-off and jet-on conditions. Consider the analogy suggested by the title of this paper whereby minimization of the stopping distance of an automobile on wet pavement requires a balance of brake-on and brake-off. The purpose of this pulsing (tapping the brake) is designed to maximize the time that the coefficient of static friction is greater than the coefficient of kinetic friction. Anti-lock braking systems are designed to maximize this effect; they recognize that if the pulsing duration is too long, then the wheels will lock and slide. In this application, the jet-on condition is the brake. If the brake is constant, then the plume tends to act like an aerospike, the pressure component of drag is reduced, and the vehicle slices (slides) through the atmosphere. If the brake is off, then the interaction of the vehicle with the atmosphere is processed by a strong shock and total pressure losses are most severe. The ability to study a large matrix of simulations is expected to yield insights into the selection of an optimal pulsing frequency (if one exists) and a nozzle geometry to maximize braking efficiency during hypersonic entry.

\section{Configuration C1, Jet Off}

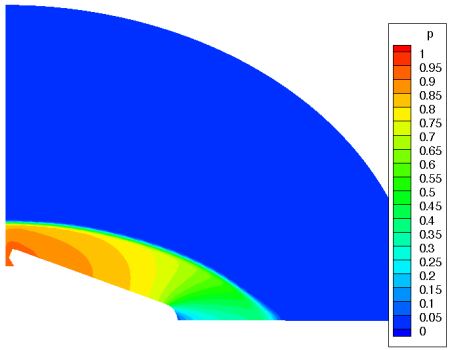

(a) $\operatorname{pressure} / \rho_{\infty} V_{\infty}^{2}$

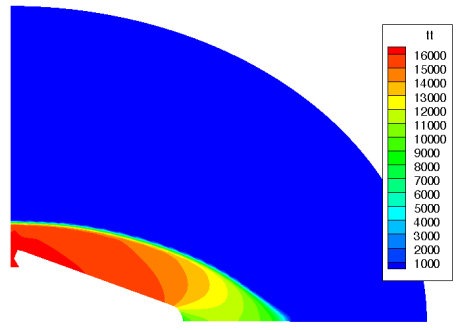

(b) temperature, $\mathrm{K}$

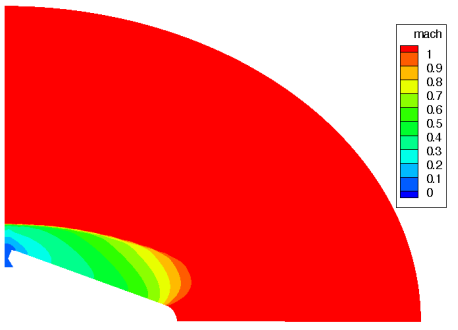

(c) Mach number

Figure 3. Contour plots after 20 flow times for a jet-off simulation.

A jet-off simulation is run in a time accurate formulation for 30 flow times (30,000 time steps). The simulation is initialized with uniform flow and attains a steady state after 10 flow times. Results are presented in Fig. 3 to enable comparisons to equivalent plots and establish a baseline for the constant jet and pulsed jet simulations to follow. Mach number is plotted from 0 to 1 to enable quick identification of the subsonic domain and any supersonic domains that are colored red in the figures. There were no Helmholtz oscillations ${ }^{15}$ observed over the open nozzle cavity with this grid resolution and time step. The drag coefficient, $C_{D}$, equals 1.487 . 


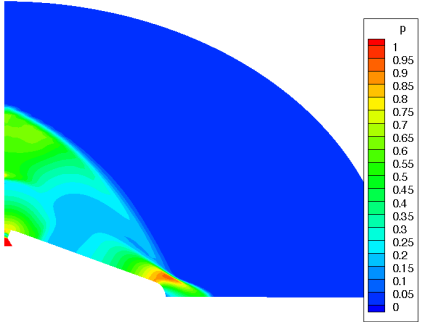

(a) pressure $/ \rho_{\infty} V_{\infty}^{2}, 57.2$ flow times

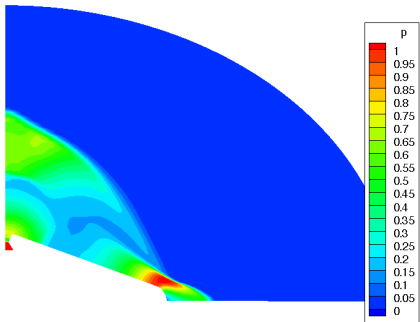

(d) pressure $/ \rho_{\infty} V_{\infty}^{2}, 58.6$ flow times

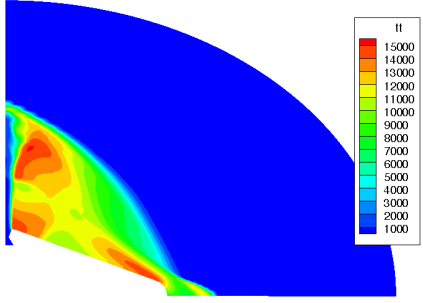

(b) temperature (K), 57.2 flow times

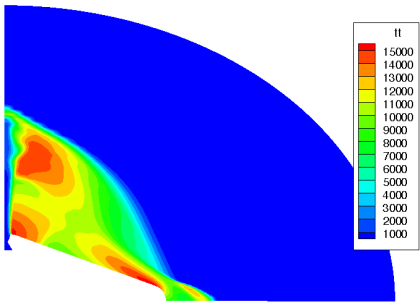

(e) temperature (K), 58.6 flow times

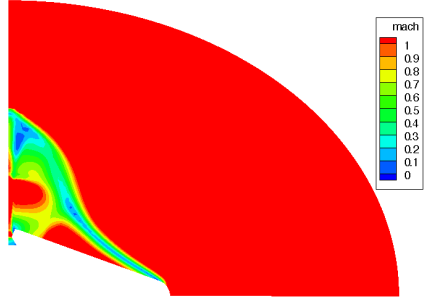

(c) Mach number, 57.2 flow times

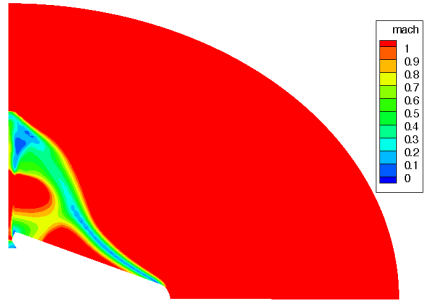

(f) Mach number, 58.6 flow times

Figure 4. Contour plots for constant jet simulation showing shock layer distributions where pressure near the shoulder is at a minimum (57.2 flow times) and at a maximum (58.6 flow times).

The jet-on simulation (constant, without pulsing) exhibits unsteady interaction with the shock layer and so must be simulated in a time accurate manner. A total of 60 flow times in 60,000 frames have been recorded. Figure 4 provides examples of pressure, temperature, and density contours in the shock layer at times when pressure over the shoulder is a minimum (57.2 flow times) and maximum (58.6 flow times). The embedded red zones in the Mach number contour indicate supersonic flow in a clockwise direction, driven by the jet plume and high pressure at the shoulder. Though heating is not computed, the temperatures over the surface are generally lower than for the jet-off condition. The drag coefficient history in Fig. 5 shows an oscillating response with a period approximately equal to 2.45 flow times after a set-up time of approximately 20 flow times. More recent simulations at higher plenum pressures and temperatures (to be shown later) exhibit the same trends but indicate peak pressures approximately three times larger than the post normal shock total pressure that periodically spill across the shoulder. In these cases, instantaneous drag coefficient peaks at $13 \%$ greater than the jet-off value, but the average drag coefficient is approximately $17 \%$ lower than the jet-off value.

The average drag coefficient beyond 30 flow times in Fig. 5 is significantly less than the jet-off simulation value of 1.487. Even though the pressure approaching the shoulder with the constant jet is larger than the jet-off simulation, the pressure within the inner $70 \%$ of the radial extent of the vehicle is larger for the jet-off condition. The reason for this lower pressure appears to be caused by the supersonic, reverse flow noted previously. This result is, of course, opposite to the stated objective - a net increase in drag. Before 
considering perturbations to these conditions the pulsed jet condition is reviewed in the next section.

\section{Configuration C1, Jet On, Pulsed}

A total of 50 flow times in 50,000 frames have been recorded for the pulsed jet condition. Figure 6 provides examples of pressure, temperature, and density contours in the shock layer at times when pressure over the shoulder is a minimum (41.4 flow times) and maximum (46.0 flow times). The embedded red zones in the Mach number contour indicate supersonic flow in a clockwise direction, driven by the jet plume and high pressure at the shoulder. The extent of supersonic flow over the vehicle surface is significantly lower than calculated for the constant jet case in Figs. 4e and 4f. The drag coefficient history in Fig. 7a shows an oscillating response period approximately equal to 1.2 flow times after a set-up time of approximately 20 flow times. Recall that the pulse frequency is exactly two flow times. A larger period is superposed on this response, showing minimums at approximately 20,30, 40, and 50 flow times. A subsequent test for a pulsed jet with a period of 10 flow times is shown in Fig. 7(b). There is no advantage observed here in the time-averaged drag from setting the pulse frequency corresponding to the larger period defined in Fig. 7(a).

The average drag coefficient beyond 20 flow times in Fig. 7 is greater than the constant jet result (Fig. 5) but still less than the jet-off simulation $\left(C_{D}=1.487\right)$. As noted previously in the constant jet simulation, the pressure approaching the shoulder with the pulsed jet is larger than the jet-off simulation, but the pressure within the inner $70 \%$ of the radial extent of vehicle is larger for the jet-off condition. It appears that strong reverse flow over substantial portions of the vehicle surface overwhelm the effect of high pressure near the shoulder for this parameter set.

In this example, the pulsed jet provides moderately better drag than the constant jet, but both jet conditions produce less drag than the jet-off condition. The pulsed jet appears to be a less-effective aerospike than the constant jet in this case with no substantial difference in the recovered pressure that is sought at the shoulder. Based on this result, perturbations in nozzle shape and plenum conditions that inject less gas into the recirculation region will be considered.

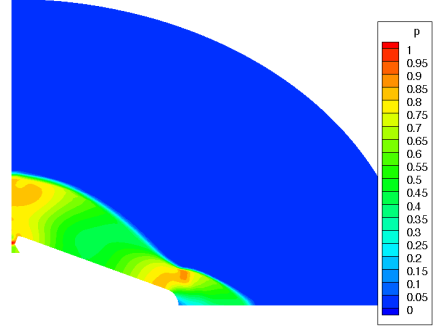

(a) pressure $/ \rho_{\infty} V_{\infty}^{2}, 41.4$ flow times

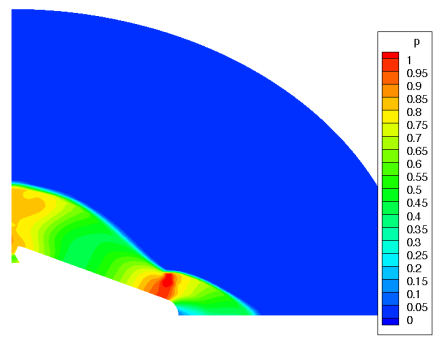

(d) pressure $/ \rho_{\infty} V_{\infty}^{2}, 46.0$ flow times

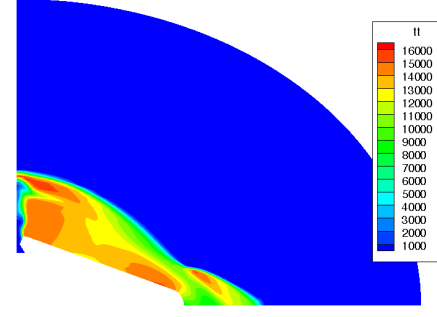

(b) temperature (K), 41.4 flow times

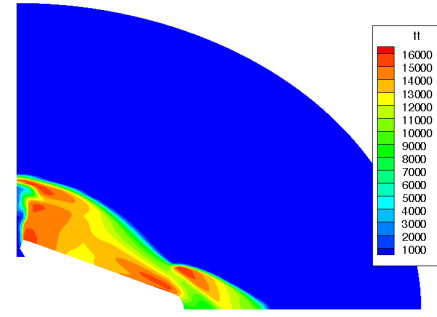

(e) temperature $(\mathrm{K}), 46.0$ flow times

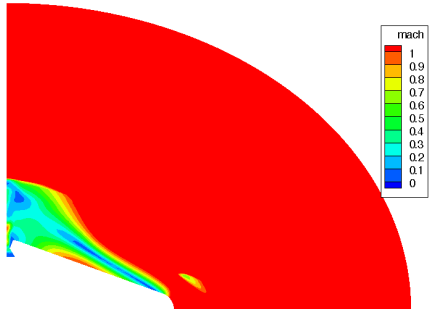

(c) Mach number, 41.4 flow times

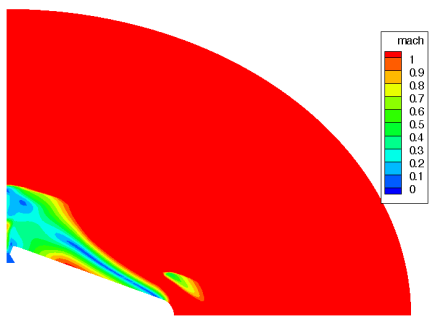

(f) Mach number, 46.0 flow times

Figure 6. Contour plots for pulsed jet simulation showing shock layer distributions where pressure near the shoulder is at a minimum (41.4 flow times) and at a maximum (46.0 flow times). 


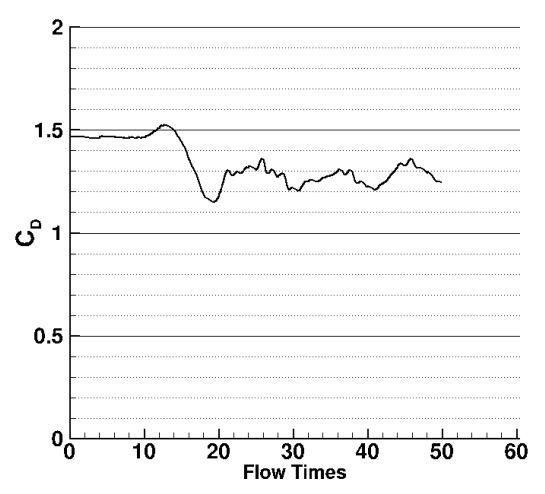

(a) pulse $=2$ flow times

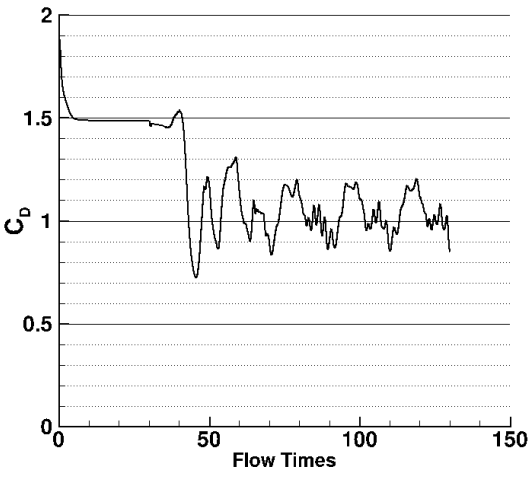

(b) pulse $=10$ flow times

Figure 7. Drag coefficient as function of flow time for the pulsed jet simulations.

\section{Configuration C2}

The temporal variation of the drag coefficient for all four conditions of conical nozzle configuration $\mathrm{C} 2$ are presented in Fig. 8. Note that condition 1 with $p_{t, p l e n u m}=5.10^{4} \mathrm{~N} / \mathrm{m}^{2}$ shows very little variation in time while conditions $2-4$ with $p_{t, \text { plenum }}=1.10^{5} \mathrm{~N} / \mathrm{m}^{2}$ show significant variation in time. Condition 1 for nozzle $\mathrm{C} 2$ yields a normal shock prior to the nozzle exit. At this condition the plume does not significantly disturb the bow shock. (In the previous configuration with a larger area ratio, $\mathrm{C} 1$, this same plenum pressure yielded a flow that separated inside the nozzle and the plume remained supersonic beyond the nozzle exit.) Conditions $2-4$ yield an over-expanded flow in which the supersonic flow in the plume pushes back on the bow shock. Increasing $T_{t, \text { plenum }}$ from $1000 \mathrm{~K}$ at condition 2 to $4000 \mathrm{~K}$ at condition 4 creates larger amplitude variations of the total drag but has negligible impact on the natural frequency of the interactions. While the drag coefficient periodically exceeds the jet off value its average value is substantially less than that baseline value.

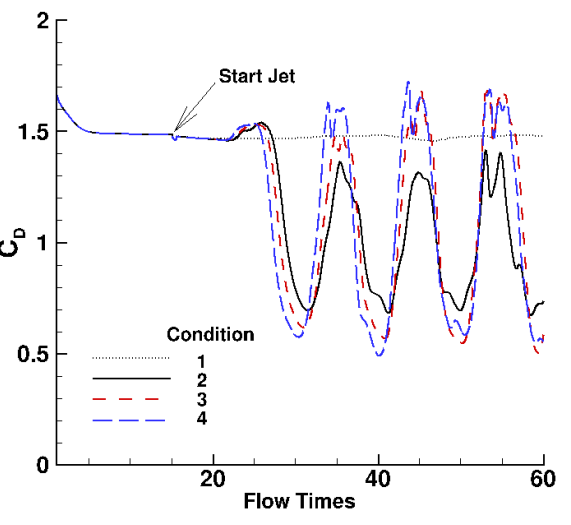

Figure 8. Drag coefficient as a function of time for a conical nozzle configuration C2 in Table 1 at conditions $1-4$ in Table 8.

\section{Configuration $C 3$}

The temporal variation of the drag coefficient for all six conditions of conical nozzle configuration C3 are presented in Fig. 9. Condition 1 in this figure (solid black line) has identical plenum conditions as configuration $\mathrm{C} 2$ at condition 2 in Fig. 8 (solid - black line). Configuration C3 has a nozzle exit radius that is $3 / 4$ of the nozzle exit radius for configuration $\mathrm{C} 2$ and a corresponding area ratio decrease equal to $9 / 16$. The smaller exit area for configuration C3 is better tuned for this flow condition, the plume penetrates the shock layer more effectively (see Fig. 10), and pressure peaks at the shoulder are somewhat larger in configuration C3 than in C2. Still, the average drag coefficient over time is less than that for the jet off condition. As seen earlier in Fig. 4, when higher than normal pressures are recovered at the shoulder, lower than normal pressures are encountered upstream, associated with high speed reverse flow. The various jet pulsing strategies cause only minor change to the baseline periodicity, approximately 9.5 flow times. Note that this periodicity is approximately four times larger than reported earlier for configuration C1. None of the pulsing strategies suggest the existence of any resonant condition that substantially improves the net drag. 


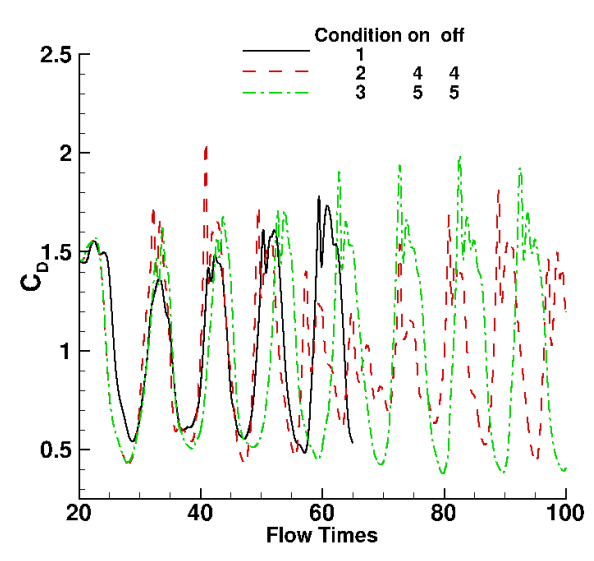

(a) Conditions 1-3

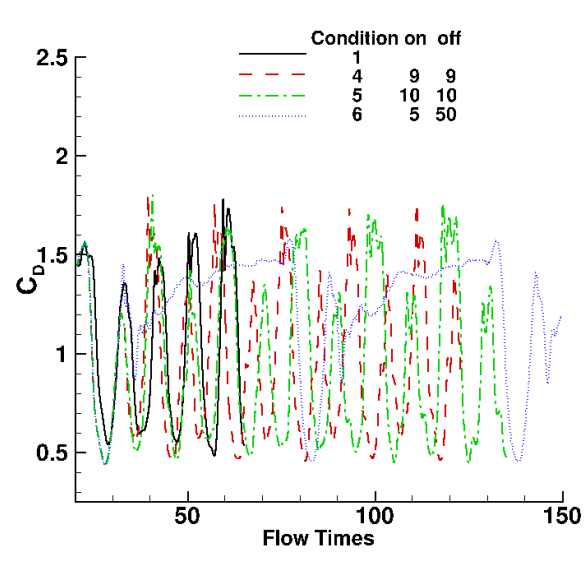

(b) Conditions 1,4-6

Figure 9. Drag coefficient as function of flow time for Configuration C3 with different pulse schedules.

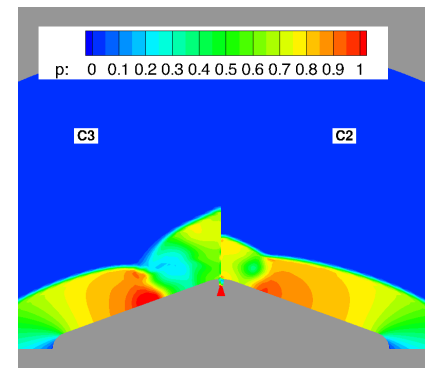

(a) 20 flow times

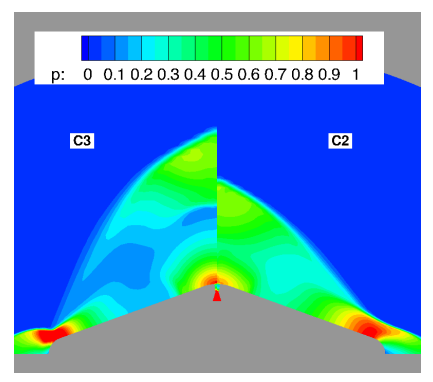

(d) 35 flow times

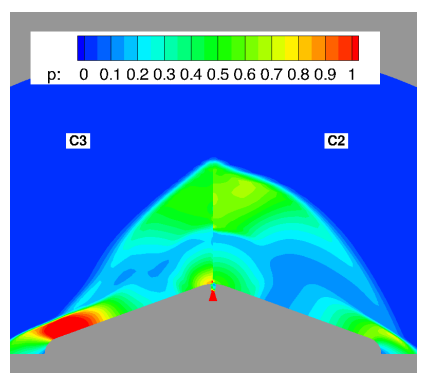

(g) 50 flow times

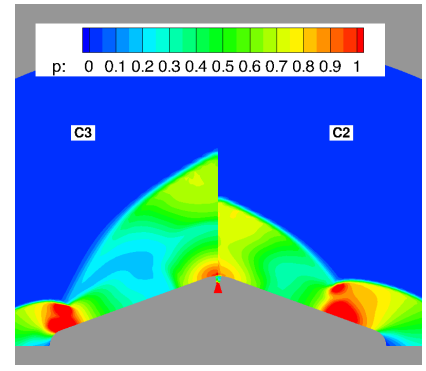

(b) 25 flow times

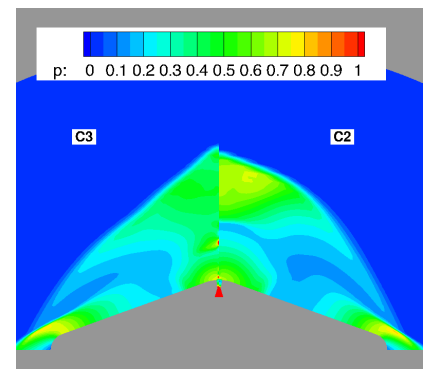

(e) 40 flow times

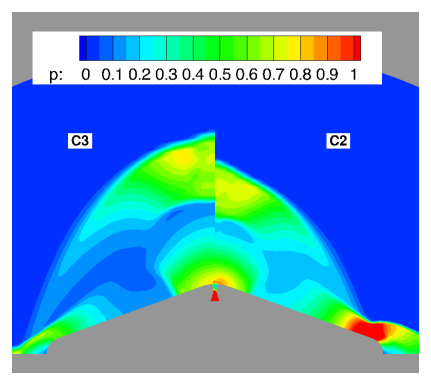

(h) 55 flow times

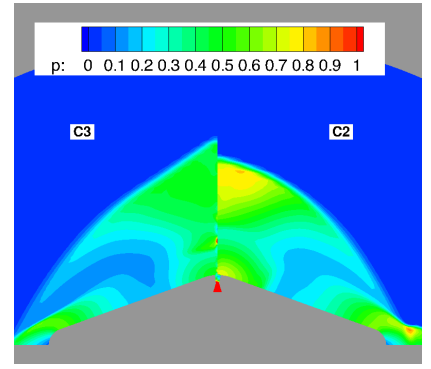

(c) 30 flow times

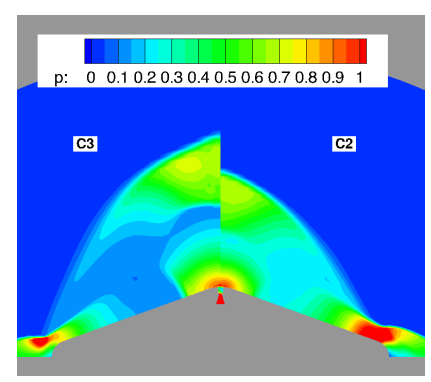

(f) 45 flow times

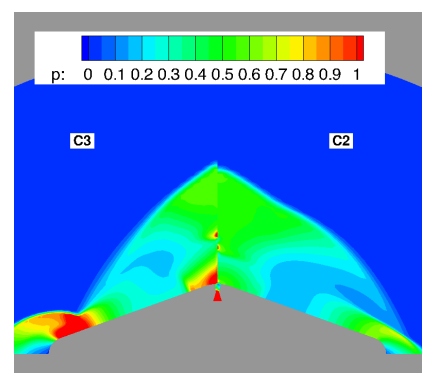

(i) 60 flow times

Figure 10. Pressure contour plots for steady jet simulation comparing configurations C2 (right) and C3 (left) with $p_{t, \text { plenum }}=1.10^{5} \mathbf{N} / \mathbf{m}^{2}$ and $T_{t, \text { plenum }}=1000 \mathbf{K}$. In both simulations, the jet is turned on at $t=15$. 


\section{Configuration $\mathrm{C}_{4}$}

In this series of simulations the nozzle exit area is further reduced to see if a thinner plume shape can produce more effective interactions with the shock layer. In Fig. 11a, plenum conditions $1-3$ repeat conditions $2-$ 6 of the previous example - with varying jet pulse schedules. As may be expected, the smaller area ratio in configuration 4 requires larger plenum pressures to maintain nozzle effectiveness. Effects of plenum pressure are captured in Fig. 11b in which conditions $4-8$ gradually increase the plenum pressure with a pulse schedule of 0.5 flow times on and 5 flow times off. These short bursts of propellant, with longer times between bursts, tend to diminish the amplitude of the interaction for conditions 6 and 7 . Conditions $8-$ 15 gradually increase the time between pulses. The most effective of these eight cases for maintaining a higher drag coefficient are shown in Fig. 11c. These three most effective cases are ones in which the time between pulses most closely match the natural frequency of the unsteady interaction for the constant jet firing conditions. Condition 16 doubles the jet on schedule of condition 12 (Fig. 11d), but maintains the time between pulses.

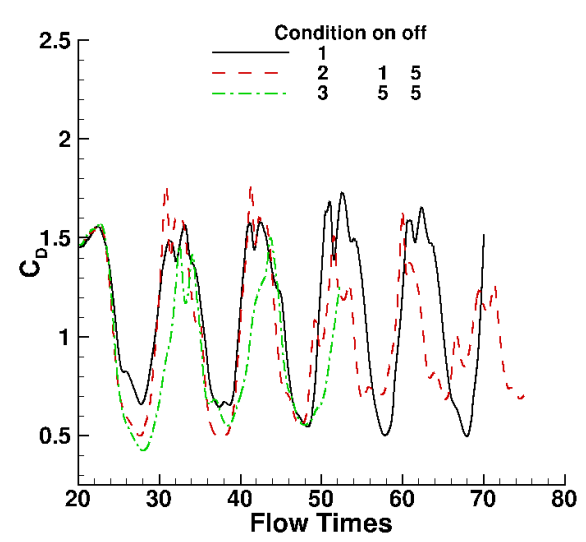

(a) Conditions 1-3, vary pulse duration

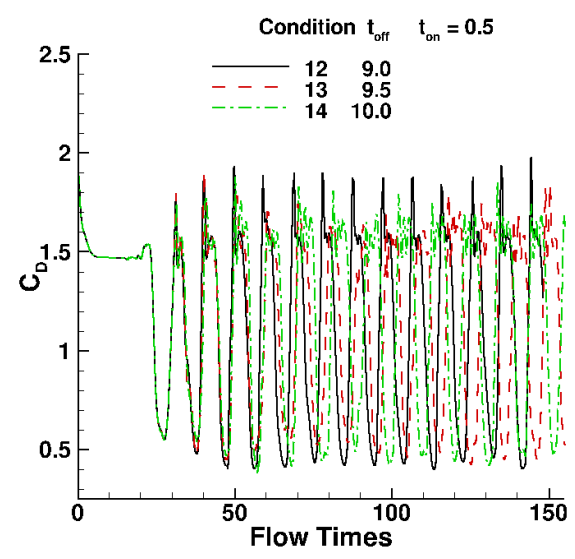

(c) Conditions 12-14, vary time pulses

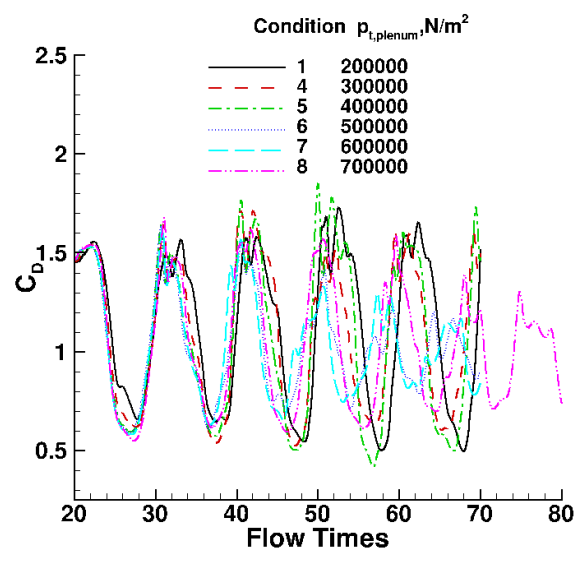

(b) Conditions 1,4-8, vary plenum pressure

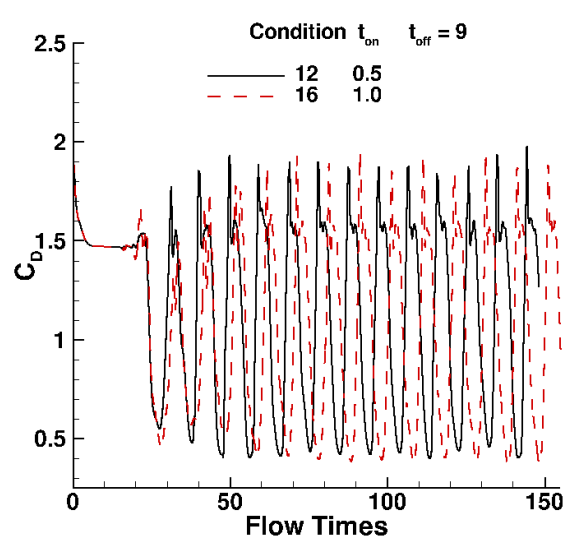

(d) Conditions 12,16, vary pulse duration

Figure 11. Drag coefficient as function of flow time for Configuration C4 with different pulse schedules.

While none of the pulsing strategies identify the existence of a resonant condition that improves the average net drag, conditions $12-14$ do exhibit a resonant response that tend to maximize the amplitude of the net variation in drag. The shock layer flow at the maximum and minimum locations of drag in time are presented in Fig. 12. The pressure contours, $p / \rho_{\infty} V_{\infty}^{2}$, are set to vary between 0 and 3 . The Mach contours are set to vary between 0 and 1 to accentuate regions of subsonic and supersonic flow. At peak conditions (136 and 146 flow times), the stream tube approaching the shoulder is processed by an oblique shock and 
peak pressure on the surface exceeds three times the stagnation pressure. At minimum conditions, that same stream tube is processed by a strong shock and pressure levels over the entire body are lower than the conditions with no jet firing.

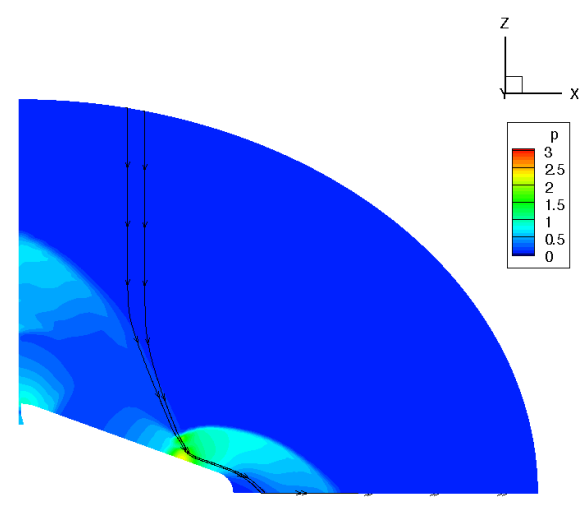

(a) pressure, 136 flow times

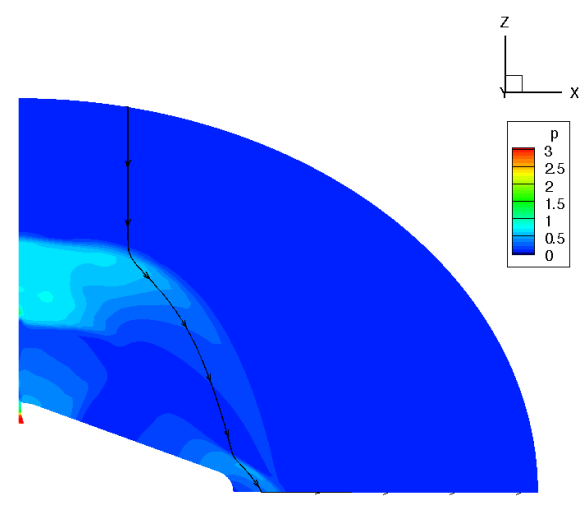

(c) pressure, 141 flow times

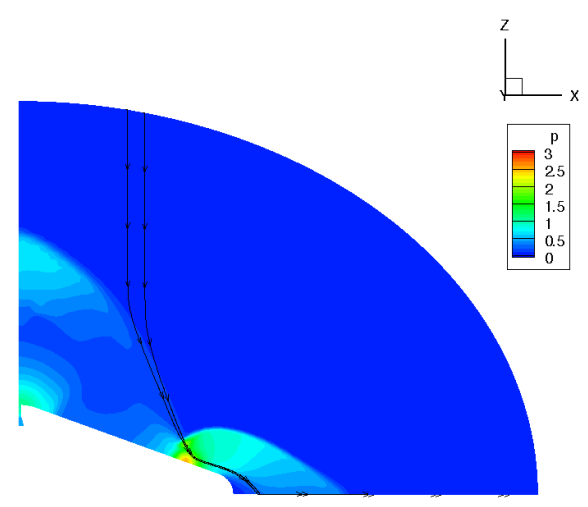

(e) pressure, 146 flow times

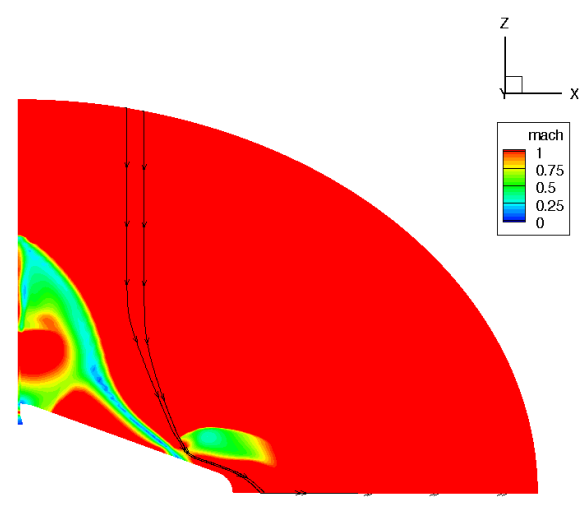

(b) Mach number, 136 flow times

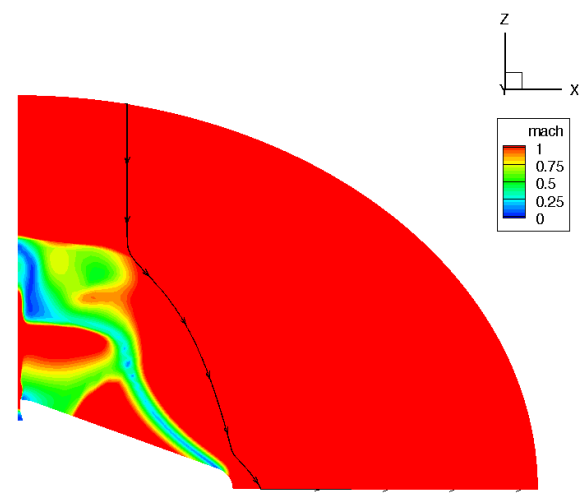

(d) Mach number, 141 flow times

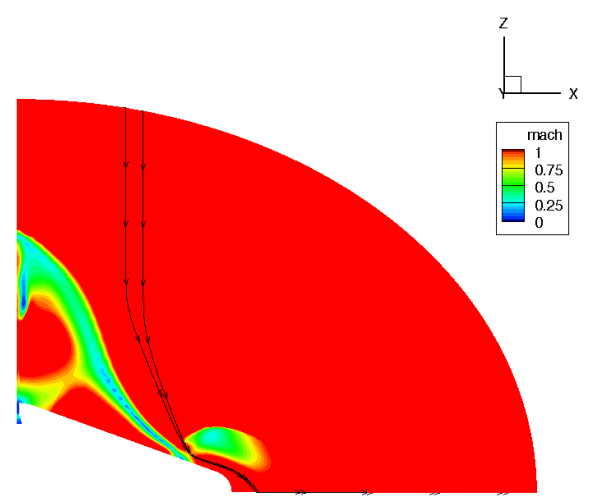

(f) Mach number, 146 flow times

Figure 12. Shock layer profile for configuration $\mathrm{C} 4$ at condition 12 where the net drag is near a local maximum at $t=136$ and 146 flow times and where it is near a minimum at $t=141$ flow times. 


\section{B. Annular Nozzle}

The plenum conditions for all of the annular nozzle configurations are defined in Table 9 . The nozzle flow has a near normal shock at the exit for configuration A1 at condition 1 and shows approximately $2 \%$ decrease in drag. Condition 2 doubles the plenum pressure and the supersonic annular jet is turned outward; the plume never directly impacts the bow shock though the entire shock layer grows in response to the mass added to the system. The resultant flow is very weakly unsteady and shows a net decrease in drag of approximately $2.5 \%$. None of the annular nozzle configurations or pulse schedules show any benefit in creating conditions to increase drag. Nevertheless, some interesting interactions are observed.

Table 9. Annular Nozzle Test Cases

\begin{tabular}{|c|c|c|c|c|c|}
\hline Geometry & Condition & $\begin{array}{c}p_{t, \text { plenum }} \\
\text { Plenum Total } \\
\text { Pressure, } \mathrm{N} / \mathrm{m}^{2}\end{array}$ & $\begin{array}{c}T_{t, \text { plenum }} \\
\text { Plenum Total } \\
\text { Temperature, } \mathrm{K}\end{array}$ & $\begin{array}{c}t_{\text {on }} / t_{\text {flow }} \\
\text { Pulse Duration }\end{array}$ & $\begin{array}{c}t_{o f f} / t_{f l o w} \\
\text { Time Between Pulses }\end{array}$ \\
\hline $\mathrm{A} 1$ & 1 & $1.0010^{5}$ & 1000 & on & NA \\
\hline $\mathrm{A} 1$ & 2 & $2.0010^{5}$ & 1000 & on & $\mathrm{NA}$ \\
\hline A2 & 1 & $2.0010^{5}$ & 1000 & on & $\mathrm{NA}$ \\
\hline $\mathrm{A} 2$ & 2 & $2.0010^{5}$ & 10000 & on & NA \\
\hline $\mathrm{A} 2$ & 3 & $2.0010^{5}$ & 1000 & 2 & 2 \\
\hline $\mathrm{A} 2$ & 4 & $2.0010^{5}$ & 1000 & 5 & 5 \\
\hline $\mathrm{A} 2$ & 5 & $2.0010^{5}$ & 1000 & 10 & 10 \\
\hline $\mathrm{A} 2$ & 6 & $2.0010^{5}$ & 100 & 2 & 2 \\
\hline $\mathrm{A} 2$ & 7 & $2.0010^{5}$ & 100 & 5 & 5 \\
\hline $\mathrm{A} 2$ & 8 & $2.0010^{5}$ & 10000 & 5 & 5 \\
\hline $\mathrm{A} 2$ & 9 & $4.0010^{5}$ & 1000 & on & NA \\
\hline A3 & 1 & $2.0010^{5}$ & 1000 & 5 & 5 \\
\hline $\mathrm{A} 3$ & 2 & $2.0010^{5}$ & 1000 & 1 & 5 \\
\hline $\mathrm{A} 3$ & 3 & $2.0010^{5}$ & 1000 & .5 & 5 \\
\hline
\end{tabular}

If the jet firing is steady through the annular jet A2, then the flow field tends to be steady as well, with only very slight fluctuations in the position of the dividing streamline separating the jet effluent from the incoming flow. This steady response may prove to be beneficial in creating a relatively low temperature buffer gas over the surface to protect the vehicle from high heating rates, as seen in Fig. 13. While there is a slight decrease in drag, this configuration could provide a simple method for protecting the heat shield for high speed entries where the plenum total temperature is less than the free stream total temperature.

Drag as a function of time for various annular nozzle configurations and jet pulse schedules are presented in Fig. 14. The near-steady response described in association with Fig. 13 is shown here in Fig. 14a. The variation of drag as a function of time indicates intriguing bifurcations as a function of nozzle geometry and pulse schedule in Figs. $14 \mathrm{~b}-\mathrm{d}$. Note that the drag coefficient exhibits two characteristic responses. In the

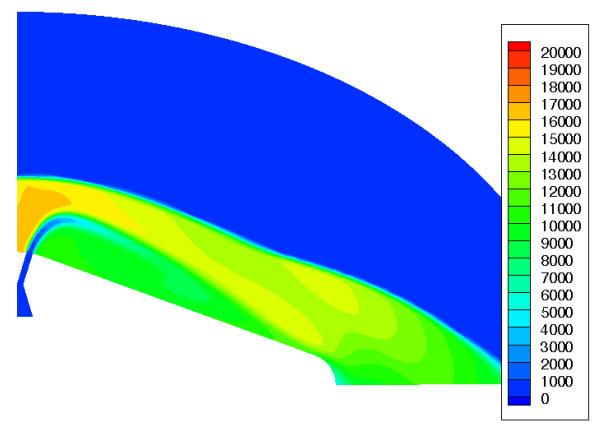
first, it tends to fluctuate around a value near 1.5 with a periodicity related to the jet pulse schedule. In the second, it tends to drop down to a value near 1 and the fluctuations have larger amplitude and appear somewhat chaotic.

These two responses may be understood by studying the sequence of frames in Fig. 15 showing temperature contours in the shock layer for configuration A2. Because the plenum total temperature is relatively 


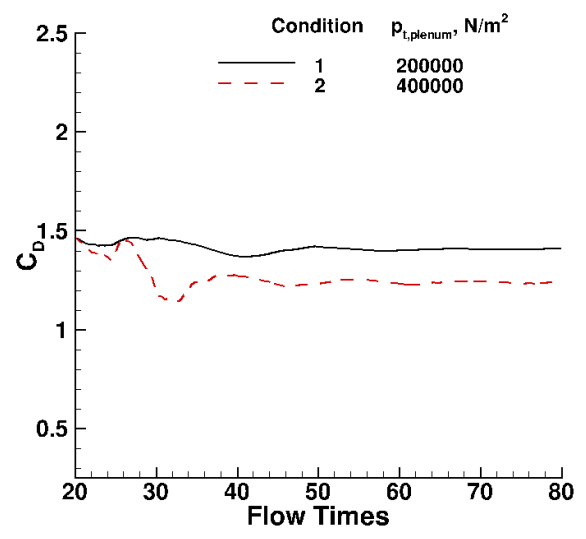

(a) Configuration A2, jet on, vary $p_{t, p l e n u m}$

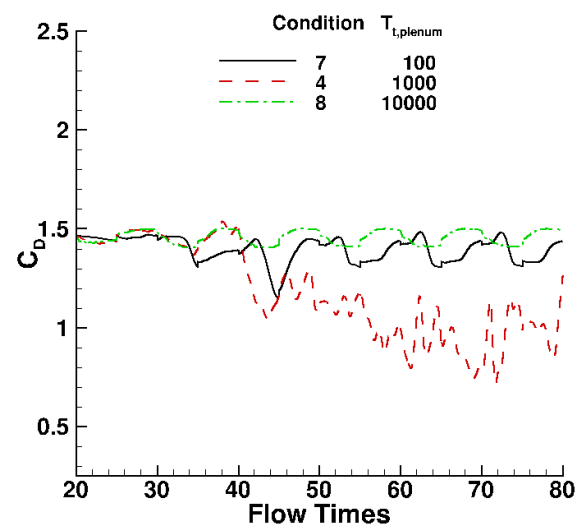

(c) Configuration A2, same pulse, vary $T_{t, \text { plenum }}$

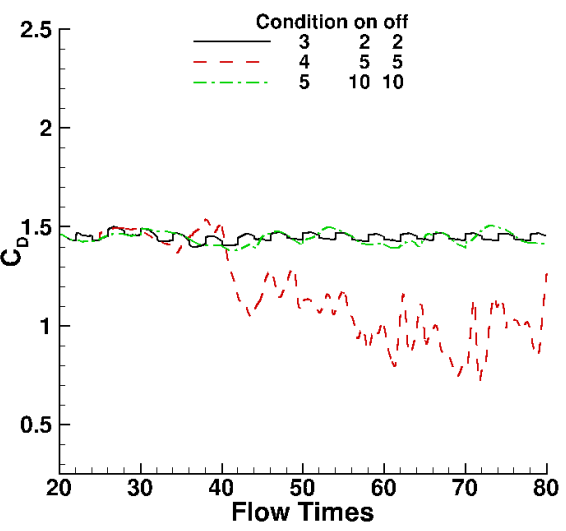

(b) Configuration A2, vary jet pulse schedule

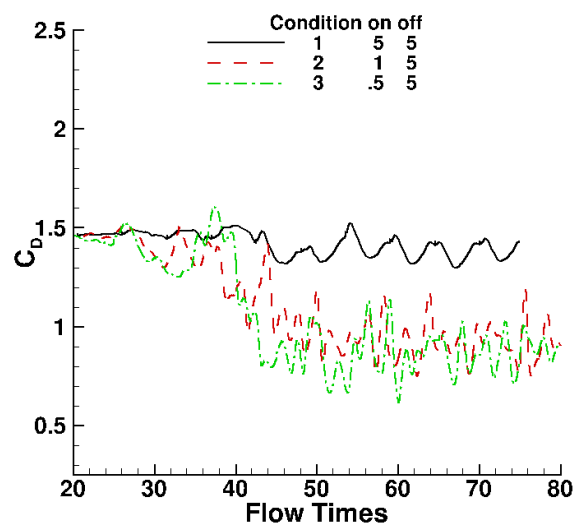

(d) Configuration A3, vary pulse-on schedule

Figure 14. Drag coefficient as function of flow time for annular nozzles.

cool, the plume shape is easily discerned with this contour. The left half of each frame shows the simulation in which the pulse schedule is five flow times on and five flow times off. This condition produces a large decrease in the average drag coefficient and large fluctuations of net drag. The right half of each frame shows the simulation in which the pulse schedule is 10 flow times on and 10 flow times off. This condition produces a low amplitude, periodic fluctuation near a drag coefficient of 1.5. At 25 flow times (Fig. 15a), both of the jets have been firing for five flow times and the solutions are identical. As the frames progress, it is evident that gas from the plume on the left collapses back toward the center of the annulus at 35 flow times while gas from the plume on the right continues to be directed downstream. As this process continues, the annular plume on the left converts to an axisymmetric plume, which is far more effective in pushing back against the bow shock. This configuration looks more like an aerospike with resultant loss in drag. The plume on the right retains its annular shape, even as the jet continues to pulse. 


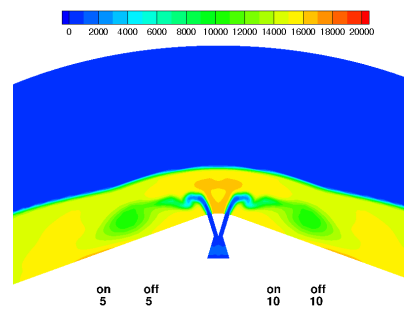

(a) 25 flow times

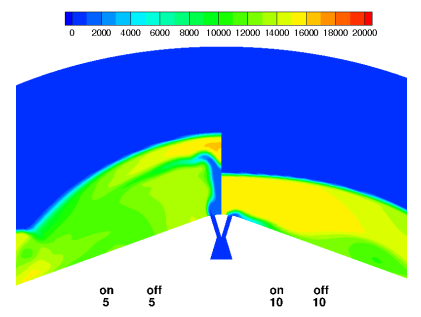

(d) 40 flow times

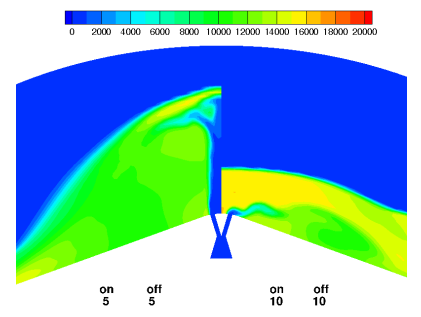

(g) 55 flow times

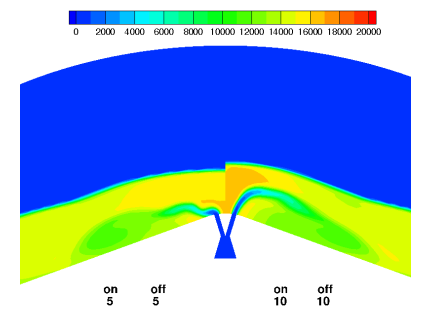

(b) 30 flow times

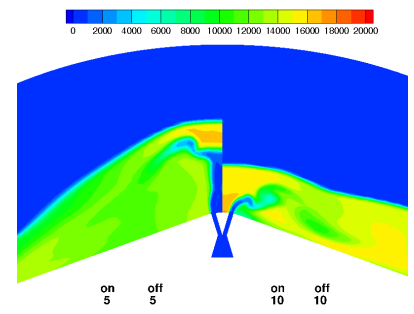

(e) 45 flow times

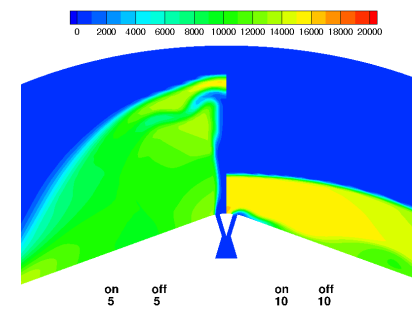

(h) 60 flow times

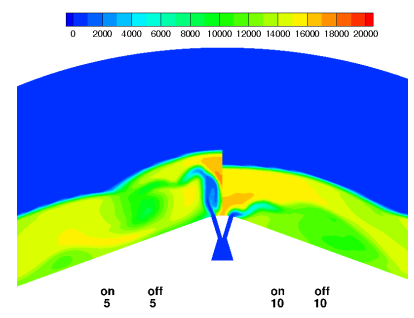

(c) 35 flow times

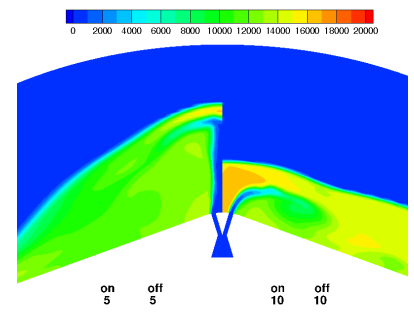

(f) 50 flow times

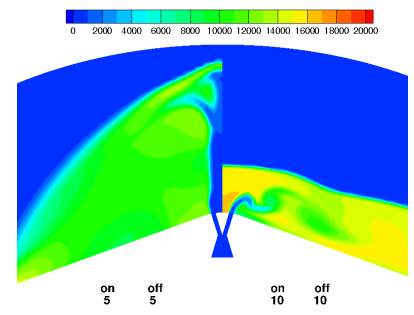

(i) 65 flow times

Figure 15. Temperature contour plots for annular jet configuration A2 comparing conditions 4 (left) and 5 (right). The pulse schedule started at 20 flow times.

\section{Symmetric Firing of Multiple Nozzles}

The plenum conditions for all of the multiple nozzle configurations are defined in Table 10. The examples here represent the initial foray into this multiple nozzle design space with intent to understand the simulation challenges. Figure 16 compares the evolution of the drag coefficient for several of these configurations, including effects of grid density and spatial order of accuracy.

Configuration N2 is identical to configuration N1 except that the nozzle throat area is reduced by a factor of two. Both of these nozzles are relatively slender (extrusion angle $\Phi_{N}=0.9$ ) and the narrow plume is easily disturbed by the interacting flow environment. As seen in Fig. 16a, the variation of the drag coefficient appears chaotic, with little indication that the throat area makes any significant difference in the response. As with previous cases, the drag coefficient occasionally exceeds the jet-off value $\left(C_{D}=1.487\right)$, but the average value is still lower and shows no indication of potential value for EDL applications. One could probably increase the plenum pressure to create a more stable plume, but this condition is already at $p_{t, \text { plenum }}=20$ bars.

It is interesting to note that simply reducing the spatial order of accuracy from second- to first-order suppresses the chaotic response. Configuration N1 at condition 1 was run using the baseline, second-order, spatial discretization in Fig. 16a (solid black line) and using a first-order discretization in Fig. 16b (solid black line). The first-order simulation has a very weak, periodic response to the jet-on condition. Increasing the plenum pressure to 50 bars, again with first-order spatial discretization, produces a periodic response 
Table 10. Multi-Nozzle Test Cases

\begin{tabular}{|c|c|c|c|c|c|}
\hline Geometry & Condition & $\begin{array}{c}p_{t, \text { plenum }} \\
\text { Plenum Total } \\
\text { Pressure, } \mathrm{N} / \mathrm{m}^{2}\end{array}$ & $\begin{array}{c}T_{t, \text { plenum }} \\
\text { Plenum Total } \\
\text { Temperature, } \mathrm{K}\end{array}$ & $\begin{array}{c}t_{\text {on }} / t_{\text {flow }} \\
\text { Pulse Duration }\end{array}$ & $\begin{array}{c}t_{o f f} / t_{f l o w} \\
\text { Time Between Pulses }\end{array}$ \\
\hline N1 & 1 & $2.0010^{6}$ & 1000 & on & NA \\
\hline N1 & 2 & $2.0010^{6}$ & 5000 & on & NA \\
\hline N1, N1f & 3 & $5.0010^{6}$ & 5000 & on & NA \\
\hline N1 & 4 & $5.0010^{6}$ & 5000 & 6 & 6 \\
\hline $\mathrm{N} 2$ & 1 & $2.0010^{6}$ & 1000 & on & NA \\
\hline $\mathrm{N} 3, \mathrm{~N} 3 \mathrm{f}$ & 1 & $5.0010^{6}$ & 5000 & on & $\mathrm{NA}$ \\
\hline $\mathrm{N} 4$ & 1 & $2.0010^{5}$ & 1000 & on & NA \\
\hline N5 & 1 & $2.0010^{5}$ & 1000 & on & NA \\
\hline N6 & 1 & $2.0010^{5}$ & 1000 & on & NA \\
\hline N7 & 1 & $2.0010^{5}$ & 1000 & on & $\mathrm{NA}$ \\
\hline
\end{tabular}

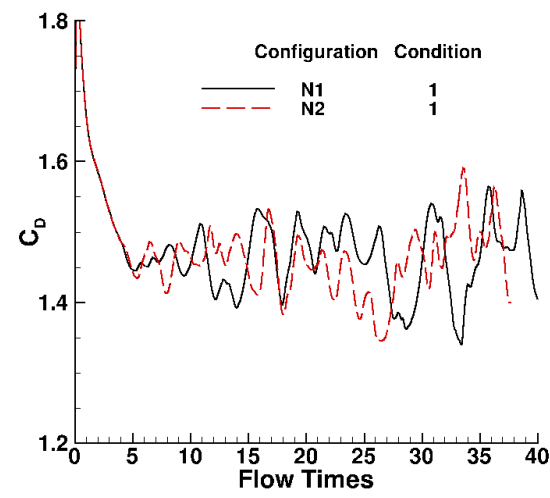

(a) Configurations N1 and N2 with different throat areas.

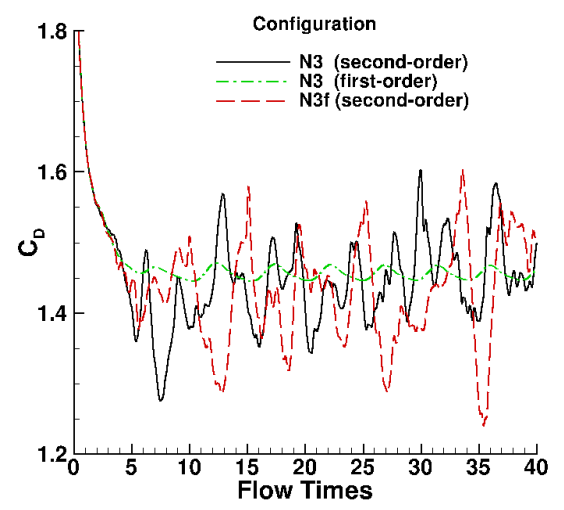

(c) Effects of grid refinement and spatial order for configuration N3.

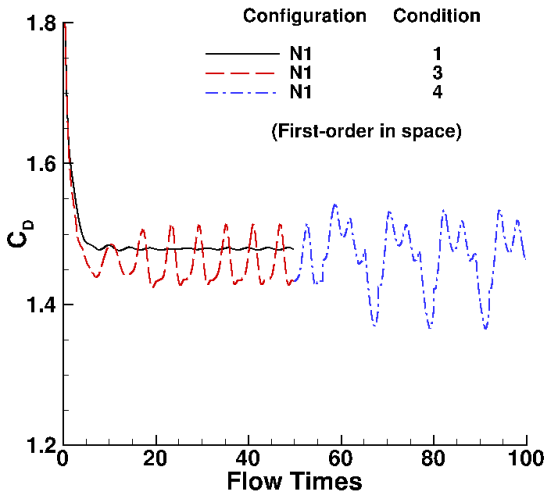

(b) Configuration N1 with different plenum conditions.

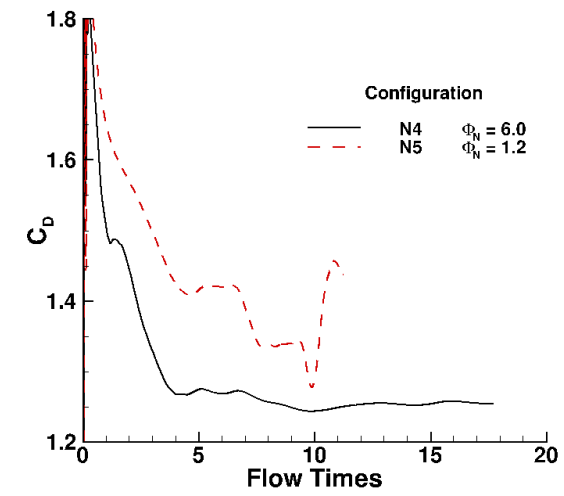

(d) Effects of nozzle extrusion angle $\Phi_{N}$ (width) at $r_{n o z}=0.6 \mathrm{~m}$.

Figure 16. Drag coefficient for several symmetrically placed, multi-nozzle configurations.

with larger amplitude in Fig. 16b (red dash line). Pulsing the jet (condition 4) produces a more complex, periodic response with still larger amplitude (blue dash-dot line). Again, the drag coefficient for all of these 
cases is less than the jet-off value and shows no indication of potential value for EDL applications.

The exploration of effects of spatial order and grid refinement are continued in Fig. 16c. Configuration N3 has a much smaller throat radius $\left(r_{\text {throat }}=0.0002 \mathrm{~m}\right)$ than $\mathrm{N} 1$ or $\mathrm{N} 2$. Plenum conditions for this test are 50 bar total pressure and $5000 \mathrm{~K}$ total temperature. The first-order accurate simulation has a small amplitude, periodic response (green dash-dot line) while the second-order accurate simulation (black solid line) indicates a larger amplitude, chaotic response. This qualitative comparison is exactly the same as seen previously for configuration N1. Doubling the number of cells for this case (configuration N3f) with second-order accurate simulation produces a similar, chaotic drag coefficient response (red dashed line). The fine grid case starts to diverge from the baseline grid case at approximately five flow times. The drag coefficient minimums tend to be lower than the baseline grid case. It is not clear if the differences are an indication of a lack of grid convergence, insufficient temporal convergence in the sub-iterations, or an extreme sensitivity to initial conditions. If the issue is extreme sensitivity when the plumes are very narrow, then it greatly diminishes the prospects of being able to sculpt a resonant interaction with controlled pulses that will increase the average drag over time.

The drag coefficient for configuration N4 (with the wide nozzle) and configuration N5 (with the slim nozzle) are presented in Fig. 16d. Recall that both nozzles are located farther downstream on the conical surface at $r_{n o z}=0.6 \mathrm{~m}$, and both conditions are for a constant jet-on. The wide nozzle result (relative to previous simulations) is predominantly steady. The slim nozzle result shows a very dynamic response, even without jet pulsing. In general, it is observed that if the plenum pressure and the plume cross section are sufficiently large, then the jet interaction with the shock layer tends to be steady. Conversely, if the plenum pressure is only slightly larger than the pressure at the exit, the jet is weak, and its interaction with the shock layer also tends to be steady. Because this investigation is looking at jets with minimum propellant mass requirements that are also strong enough to perturb the bow shock, they tend to cause unsteady interactions within the shock layer.

The simulations for configurations N6 $\left(\theta_{c}=55\right.$. deg.) and N7 ( $\theta_{c}=45$. deg.) had essentially constant drag response to the jet-on condition with drag coefficients equal to 1.396 and 0.967 , respectively. Both of these configurations positioned the nozzle exit downstream of the sonic line on the nose. These two cases exhibited a very high frequency oscillation (less than 0.1 flow times) with amplitude $\Delta C_{D}<0.001$. They could be run as a steady simulation and would converge to machine zero after freezing the flux limiter. These results indicate that jets blowing into an inviscid, supersonic external flow have a greater tendency to remain steady, as compared to jets blowing into an inviscid, subsonic external flow. The presence of a boundary layer in a viscous flow is expected to modify this observation.

The chaotic response of the drag coefficient seen in Fig. 16c can be better understood by studying snapshots of the interaction for configuration N3f in Fig. 17 with jet-on (no pulsing of the plenum). An isosurface of temperature equal to $5000 \mathrm{~K}$ effectively captures the surface of the bow shock at Mach 20 (perfect gas) as well as the edge of the jet plume surrounding a core of gas with temperature less than the plenum total temperature. Surface contours in this figure also show the corresponding surface pressure with contour values in red greater than stagnation pressure for the jet-off condition. The time between each frame, 0.5 flow times, corresponds to 500 time steps in the simulation. Note that the jet plume does not extend far into the shock layer at 88.0 flow times. It is recovering from a fluctuation where the local pressure was large and the plume was effectively bent over. At 89.0 flow times, the plume begins to recover; it becomes straight and penetrates the bow shock from 89.5 flow times to 91 flow times. The plume begins to collapse again at 91.5 flow times. The plume and the bow shock are almost fully collapsed at 93 flow times, after which the cycle begins again (although not in an exactly periodic manner).

The bow shock fluctuations indicate distinct ridge lines corresponding to the action of the plume pushing outward, creating its own shock system that evolves with plume unsteadiness. These ridges indicate locations where the remnants of the plume shock intersect the larger bow shock of the vehicle. Shock angles at these ridges tend to be most oblique relative to the incoming flow, and total pressure losses are consequently smaller than losses encountered across the unperturbed bow shock. Sweeps of red color on the surface, indicative of pressures exceeding $p_{t, 2}$ across a normal shock, roughly follow the movement of these bow shock ridge lines, most evident starting at 90.5 flow times in Fig. $17 \mathrm{f}$ and continuing to 92.0 flow times in Fig. 17i.

It is encouraging to see large swaths of high pressure across the surface; however, it is clear that the average net drag is still less than the jet-off condition. Furthermore, the chaotic nature of this surface pressure response makes this a destabilizing system; while the simulation here imposed symmetry boundary conditions on the side planes the results indicate that it is unlikely for a truly four dimensional flow (three 
space and time) to remain symmetric.

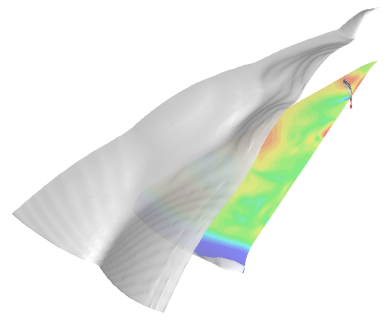

(a) 88.0 flow times

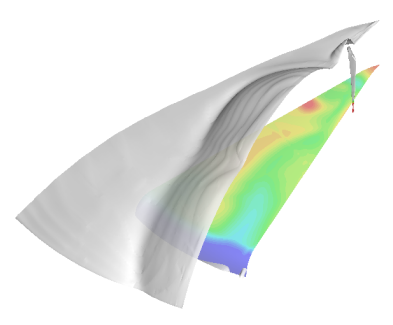

(d) 89.5 flow times

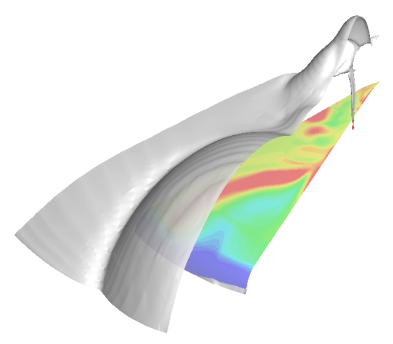

(g) 91.0 flow times

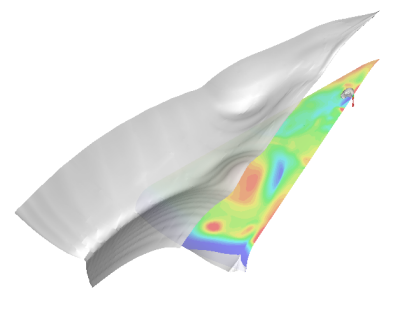

(j) 92.5 flow times
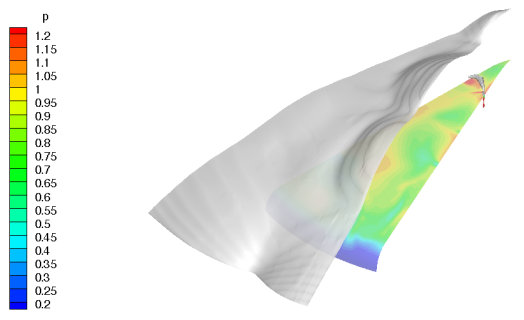

(b) 88.5 flow times
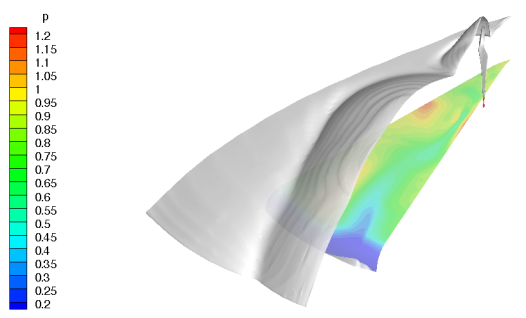

(e) 90.0 flow times
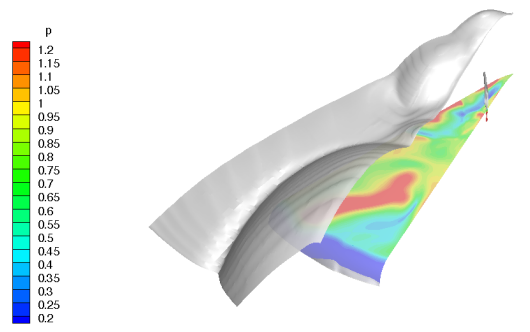

(h) 91.5 flow times
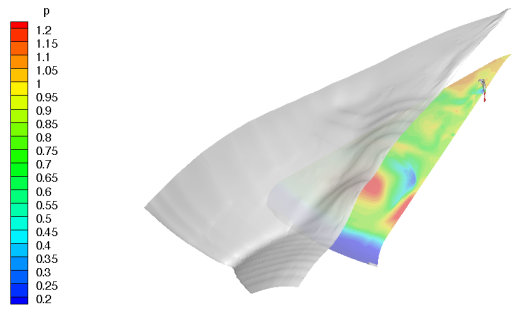

(k) 93.0 flow times
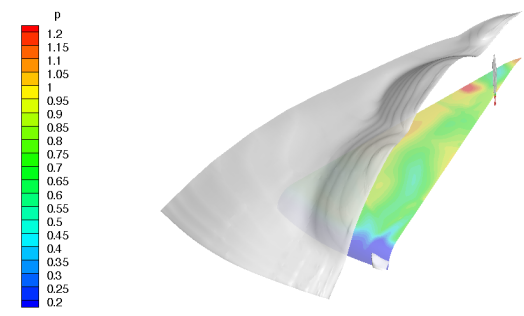

(c) 89.0 flow times
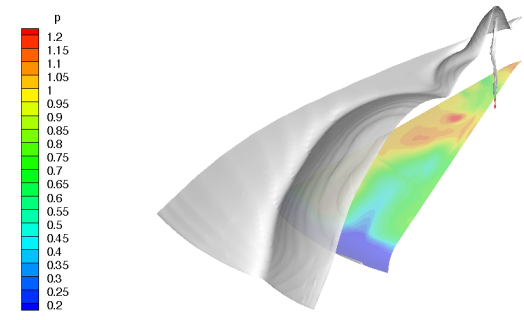

(f) 90.5 flow times
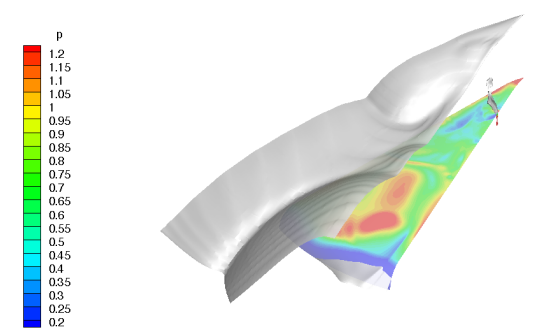

(i) 92.0 flow times
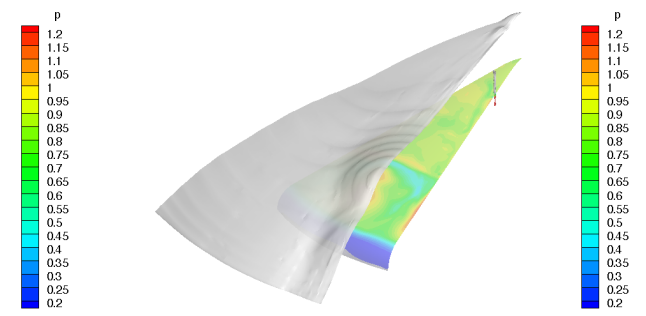

(1) 93.5 flow times

Figure 17. Iso-surfaces in gray of constant temperature equal to $5000 \mathrm{~K}$ to highlight edge of jet plume and the bow shock for configuration N3f. Surface contours of pressure highlight values larger than stagnation conditions in red. 


\section{Discussion}

The goal of this study is to better understand the conditions through which a significant increase of the drag coefficient of a planetary entry vehicle may be obtained through aerodynamic interaction of pulsed jets opposing an incoming, hypersonic flow (tapping the brake). The essential element to achieve this goal is to create conditions by which a larger fraction of the free stream total pressure may be recovered to the surface by processing stream tubes through oblique (weak) shocks rather than normal (strong) shocks. The key concept here is that aerodynamic interference is the primary source of drag. While it is certainly possible to overpower the flow field with a large retro-firing thruster (with its concomitant system mass and propellant mass requirements), we explore the possibility that significant increase in drag may be achieved at hypersonic conditions with coordinated firing of much smaller thrusters.

Optimum conditions (if they exist) must produce a plume that is strong enough to displace the bow shock to produce oblique discontinuities with propellant mass requirements that are less than competing concepts (e.g., supersonic retro-propulsion). Producing a high pressure near the outboard shoulder is not especially difficult. The fine grid simulation of configuration $\mathrm{C} 4$ presented in Fig. 2b briefly produces pressures approximately four times the stagnation value. The baseline grid simulation discussed in association with Fig. 12 briefly produces pressures in excess of three times the stagnation value. At these times, the drag coefficient is only $20 \%$ to $60 \%$ higher than the jet-off value. As noted earlier, there are two counteracting interactions that suppress an increase in the drag coefficient. First, the high pressure at the shoulder drives a supersonic, reverse flow towards the axis of the vehicle and the corresponding pressure acting on the vehicle surface is less than the jet-off pressure levels at the same locations. Second, the propellant gases roll back over the body and form a buffer layer that diverts the stream tubes that have crossed oblique shocks away from the body. Periodic shedding of these buffer gas layers prevent a sustained impingement of the higher total pressure stream tube near the vehicle's shoulder. Indeed, without periodic shedding, a recirculation zone is created with an embedded shock near the sphere-cone junction (see the sharp transition from red, supersonic flow to green, subsonic flow in Figs. 12b, d, and f). Such a shocked, recirculating condition would continually raise the entropy of a closed streamline in inviscid flow. Short pulses (0.5 to 1 flow times) with intervals on the order of the natural period of the interaction for the constant jet-on condition tend to yield the best average drag coefficient (Figs. 11c and d); however, this value is still less than the jet-off level, not to mention far below the stated goal of a factor two increase. More cases are planned for the very short pulses in conjunction with new simulation tools that better track moving discontinuities in the flow (discussed in Sec. VII). Based on results to date, it is expected that azimuthal variation must also be engaged to provide conditions where near-steady impingement at the shoulder is accommodated by three-dimensional relief of the buffering, recirculation zone.

The annular jet cases were not very effective at displacing the bow shock. Higher plenum pressures and/or properly tuned pulsing frequencies (Fig. 15) may make the annular jets behave more like a thicker, conical jet. There is no indication that such an annular configuration has any advantages over the conical configuration for the purpose of increasing the average drag coefficient. However, the buffer gas region with entrained propellant gases in Fig. 13 may provide a very effective means of reducing heating over a large surface area. A similar idea ${ }^{16}$ has been proposed previously; however, the injection did not utilize an annular nozzle and flow unsteadiness appeared to be an issue. This concept will be studied in future simulations including viscosity and multiple species.

The symmetric, multi-nozzle cases provide an additional degree of freedom for three-dimensional relief of the buffer gas layer in the azimuthal direction. There is still an imposed symmetry across the side planes of the computational domain. For the cases where the nozzle was located near the sphere-cone junction $\left(r_{n o z}=0.2 \mathrm{~m}\right)$, the flow interactions were all unsteady and chaotic. Simply running them with a first-order accurate formulation was enough to damp the interactions so that a temporally periodic interaction was obtained. Besides the location of the nozzles, the main difference in these simulations compared to the conical nozzle cases is the extra degree of freedom in the azimuthal direction and the use of rectangular cross - section nozzles rather than circular cross - section nozzles. (Circular cross sections will be added to the grid generation algorithm for future tests.) Recall, the new idea in this paper has been to look for configurations and plenum pulse schedules that induce the aerodynamic component of the interaction to produce significantly larger retro-force than the thrust component. Consequently, the search has focused on relatively slender nozzles and slender jet plumes. Configuration N1f at condition 3 (throat half-width equal to $0.002 \mathrm{~m}$ ) produces an underexpanded flow in Fig. 18a with $p_{\text {exit }} \approx 10$. (This value is in good agreement with isentropic expansion through a nozzle with the given plenum conditions.) Configuration N3f at condition 1 


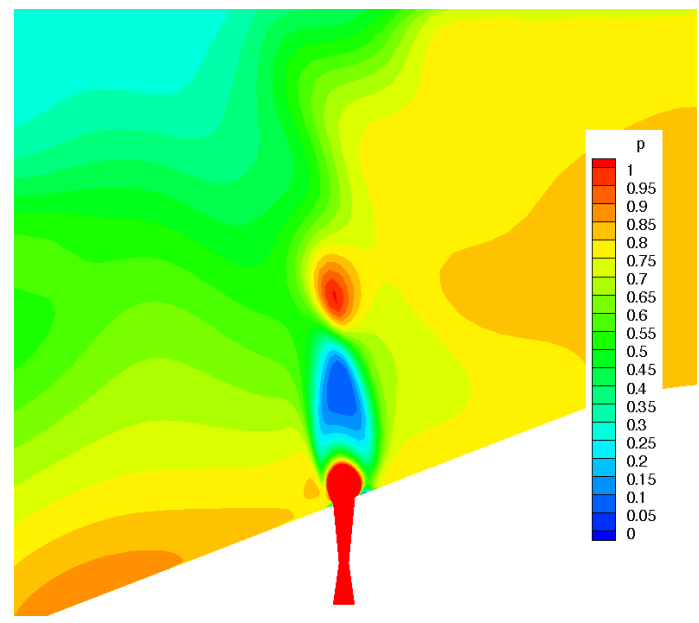

(a) Config. N1f at 26 flow times. Underexpanded

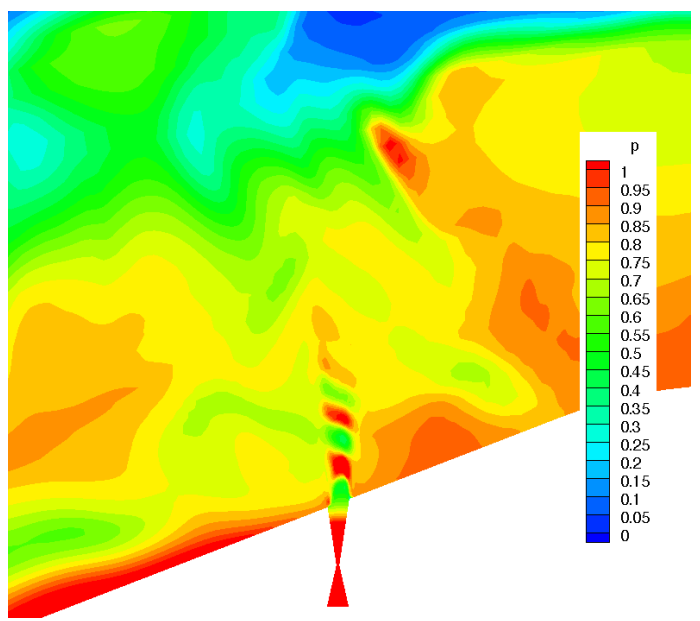

(b) Config. N3f at 90 flow times. Overexpanded

Figure 18. Pressure contours in vicinity of the nozzle.

(throat half-width equal to $0.0002 \mathrm{~m}$ ) produces an overexpanded flow in Fig. $18 \mathrm{~b}$ with $p_{\text {exit }} \approx 0.6$. (This value exceeds the expected pressure level for an isentropic expansion; the probable cause is that the throat is poorly resolved with the current grid. The effective throat half-width in this case is $0.000326 \mathrm{~m}$. The half-width of the cell below the throat is $0.000348 \mathrm{~m}$.) The plume within the first nozzle exit width of the exit plane is remarkably steady for the underexpanded flow condition. There is more unsteadiness apparent just above the nozzle exit in the overexpanded case. While steady interactions are often expected for strongly, underexpanded nozzle flows, ${ }^{17,5}$ these results suggest there is also a requirement that the diameter of the resultant plume must be at least some minimum fraction of the shock standoff distance. More simulations are needed to study variations in plume diameter and density (through variation of the total temperature in the plenum) to better understand these interactions. Chaotic unsteadiness is considered an unacceptable risk, even if the average drag can be doubled, simply because the associated pressure distributions could induce significant unsteadiness in the angle of attack.

\section{Next Generation Simulation}

Recent work, based on analyses using Walsh functions, had produced a very simple algorithm that was tested as an adjunct to FUN3D for exploring the retro-propulsion design space. ${ }^{18,19,20}$ That initial Walsh function approach focused on solving partial differential equations with finite differences (FD) as opposed to integral conservation laws. It yielded third-order accurate solutions in space and time for simple problems in one dimension, ${ }^{20}$ and it was applied to a hypersonic blunt body problem for the first time here. The FD algorithm discretizes the conservative form of the governing equations, but it is not explicitly conservative in the sense of a finite-volume formulation in which flux across cell walls is explicitly defined. The algorithm does exactly preserve a uniform flow on general, curvilinear grids.

A standard test problem, inviscid, hypersonic flow of perfect-gas air over a circular cylinder at $V_{\infty}=$ $5000 \mathrm{~m} / \mathrm{s}$ and $T_{\infty}=200 \mathrm{~K}$, was simulated with both FUN3D and the explicit, upwind, FD formulation. Simulations on this test problem showed that the FD algorithm could produce essentially the same shock layer profiles in roughly $1 / 5$ the time for a steady flow; however, the FD scheme substantially reverted to first-order spatial accuracy for time dependent flows. The FD scheme essentially damped the unsteady interactions and, consequently, it was not deployed for this investigation.

The initial exploration of the Walsh function approach for hypersonics was inspired by its fundamental use of discontinuous basis functions. The expectation was that discontinuities in hypersonic flows could be better represented by a set of basis functions that inherently preserved discontinuities. However, using Walsh function filtering ${ }^{20}$ as a limiter ignored this unique, fundamental capability. It essentially worked to force fit the Walsh function approach to shock capturing, but this approach was not more accurate than existing algorithms in FUN3D.

In contrast to filtering, feature detection provides opportunities to clearly identify discontinuities in a 
domain and to quantify the jump condition and states on either side of the discontinuity. Ultimately,an algorithm to move the discontinuity in space is desired. The concept may possibly introduce the advantages of shock fitting with less algorithm complexity. ${ }^{21}$ The ultimate challenge is to be able to detect multiple shocks, as in the shock train of Fig. 18b, and treat them as discontinuities that are rapidly created and destroyed in a chaotic flow environment. The basics of feature detection using Walsh functions in one dimension are remarkably simple and are described subsequently. Underlying details and associated proofs are beyond the scope of this paper.

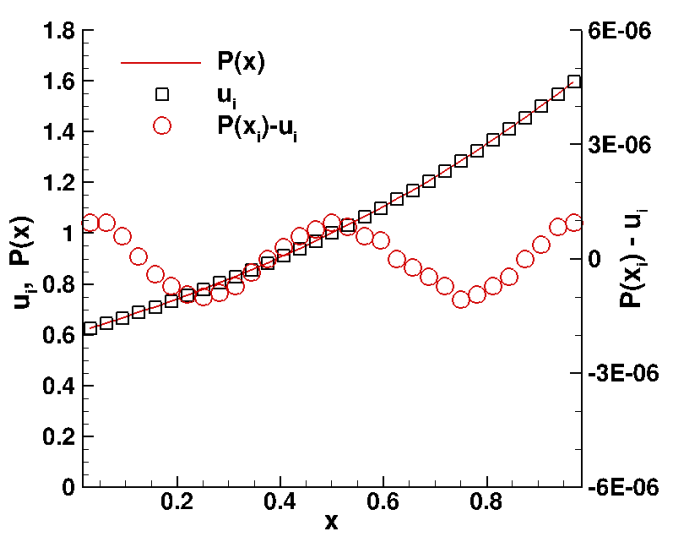

(a) Polynomial fit and error

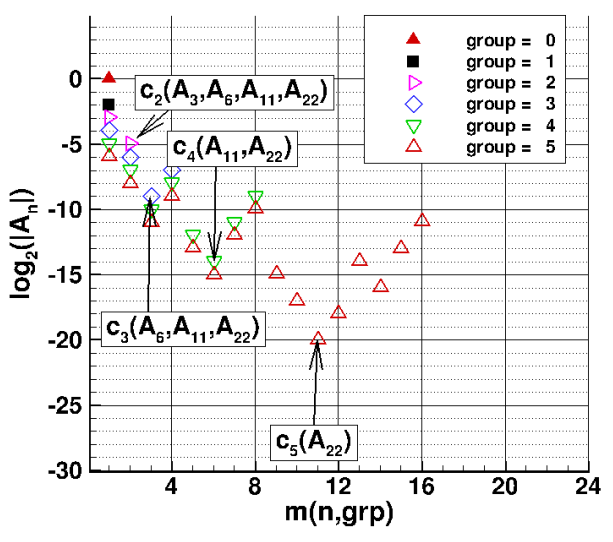

(b) Unscaled fractal fingerprint

Figure 19. Walsh function representation of smooth distribution.

First, consider the representation of a "smooth" data set $u_{i}=\exp \left(x_{i}-0.5\right)$ with $x_{i}=(i-0.5) d x$, $1 \leq i \leq 2^{p}$, and $d x=1 . / 2^{p}$. A "feature" must have context; smoothness, noise, or a discontinuity may only be discerned in the presence of some minimum number of data points. A value of $p \geq 3$ appears to be a reasonable constraint (at least eight data points) for this purpose. This sample, smooth distribution is presented in Fig.19a, and it may be represented as a series of orthogonal Walsh functions, $g_{n}(x)$, by

$$
u_{i}=\sum_{n=1}^{2^{p}} A_{n} g_{n}\left(x_{i}\right)
$$

where

$$
A_{n}=\int_{x_{a}}^{x_{b}} u(x) g_{n}(x) d x=\sum_{i=1}^{2^{p}} u_{i} g_{n}\left(x_{i}\right) d x
$$

The discrete evaluation of all $A_{n}$ is most efficiently handled by a Fast Walsh Transform ${ }^{20}$ in order $N \log (N)$ operations as opposed to the order $N^{2}$ operations required by Eq. (2). The $A_{n}$ may be organized as a fractal fingerprint, a graphical presentation that exploits the fractal nature of the Walsh function derivation in a manner that highlights repeating patterns. ${ }^{20}$ Let us assume that the discrete values of $u_{i}$ may be approximated by a polynomial, $P(x)$, of degree $p$ with a Heaviside function to account for the possibility of a discontinuity.

$$
u_{i} \approx P\left(x_{i}\right)-d_{0} H\left(x_{i}-x_{0}\right)=\sum_{m=0}^{p} c_{m} x_{i}^{m}-d_{0} H\left(x_{i}-x_{0}\right)
$$

It is observed that for a polynomial of degree $p$, there is a seed Walsh series coefficient $s(p)$ that has zero contribution from all coefficients $c_{m}$ for which $m<p$. This seed index is a member of group $p$ where group $p$ is defined as any element with index $n$ such that $2^{p-1}<n \leq 2^{p}$. Thus, in the present example showing the fractal fingerprint in Fig. 19b, the coefficient $A_{22}$ in group 5 is only a function of $c_{5}$. A simple, explicit relation enables the value of the polynomial curve fit parameter $c_{5}$ to be determined from the known value of coefficient $A_{22}$. The coefficient $A_{11}$ in group 4 is only a function of $c_{4}$ and $c_{5}$. Given that $c_{5}$ has already 
been evaluated, it is now possible to compute the value of $c_{4}$ using an explicit relation involving $c_{5}$ and $A_{11}$. In like manner, the coefficient $A_{6}$ in group 3 is only a function of $c_{3}, c_{4}$, and $c_{5}$ and the coefficient $A_{3}$ in group 2 is only a function of $c_{2}, c_{3}, c_{4}$, and $c_{5}$. Each polynomial coefficient $c_{m}$ is sequentially evaluated with an explicit relation involving Walsh series coefficients. The curve fit coefficients are captured in Table 11 and the curve fit and its error relative to the original, smooth data set are plotted in Fig.19a

Table 11. Feature Definition with Walsh Functions

\begin{tabular}{ccccccccc} 
Description & $c_{0}$ & $c_{1}$ & $c_{2}$ & $c_{3}$ & $c_{4}$ & $c_{5}$ & $d_{0}$ & $x_{0}$ \\
\hline smooth & 0.606531 & 0.606561 & 0.302784 & 0.103267 & $2.11273 \mathrm{E}-02$ & $8.44957 \mathrm{E}-03$ & 0 & 0 \\
discon. & -0.392643 & 0.590787 & 0.422066 & -0.217367 & 0.374194 & -0.128497 & 0.999498 & 0.65625 \\
noisy & -0.140803 & -3.64093 & 27.5315 & -67.2333 & 70.5019 & -26.3497 & 0.892280 & 0.65625 \\
noisy & -0.311767 & 1.06844 & -0.853875 & 0.678925 & 0 & 0 & 0.893066 & 0.65625
\end{tabular}

Next, consider the representation of a "discontinuous" data set, $u_{i}=\exp \left(x_{i}-0.5\right)-H(x-0.65625)$, as shown in Fig. 20a where the Heaviside function is zero if its argument is negative and is one if its argument is positive. The fractal fingerprint of functions with singularities in the domain are very different from the patterns seen with smooth functions. For cases where there is an abrupt jump in the distribution, the fractal fingerprint has the coefficients of the highest group number $p$ positioned equidistant from the zero axis as shown in Fig. 20b. (In some cases, the jump coincides with the step in a Walsh function of a lower group number and jump detection can be accommodated by taking the Walsh transform of the distribution shifted by one.) The magnitude of the jump can be determined by the distance of the seed coefficient $A_{s(p)}$ of that group from the axis. If the jump is less than a threshold value, then a smooth function is assumed. If the jump is greater than the threshold value, then the location of the jump is determined from the pattern of sign changes of the highest group number. Note that the location of the jump can only be determined to occur somewhere between two adjacent values of the discrete distribution $u_{i}$. Once the jump magnitude and location are determined, its effects can be subtracted from the original transform, and the algorithm for determining the coefficients of the smooth data set may be implemented. The curve fit coefficients are captured in the second row of Table 11, and the curve fit and its error relative to the original data set are plotted in Fig. 20a.

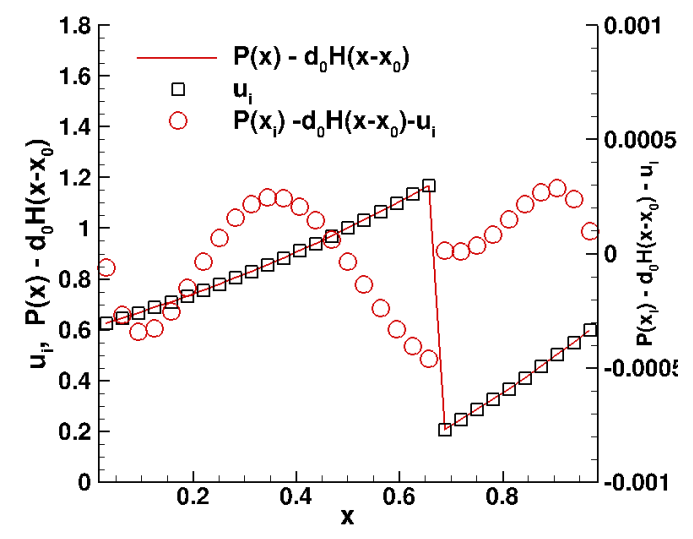

(a) Polynomial fit and error

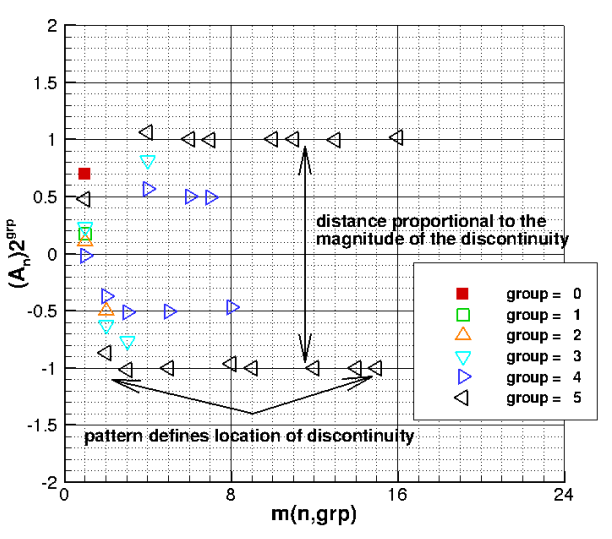

(b) Scaled fractal fingerprint

Figure 20. Walsh function representation of a discontinuous distribution.

Finally, consider the representation of the previous discontinuous data set with random noise added with amplitude 0.1. The algorithm for feature detection is unchanged. With noisy data, it is sometimes better to utilize a lower order curve fit through the data to essentially filter any attempt to capture oscillations due to noise. Examples of feature detection using a fifth-order polynomial and a third-order polynomial through $2^{5}$ 
data points are presented in Fig. 21. The corresponding curve fit constants are presented in Table 11. Work is ongoing to modify existing finite-volume formulations to utilize the feature detection capabilities defined herein for unsteady, hypersonic flows.

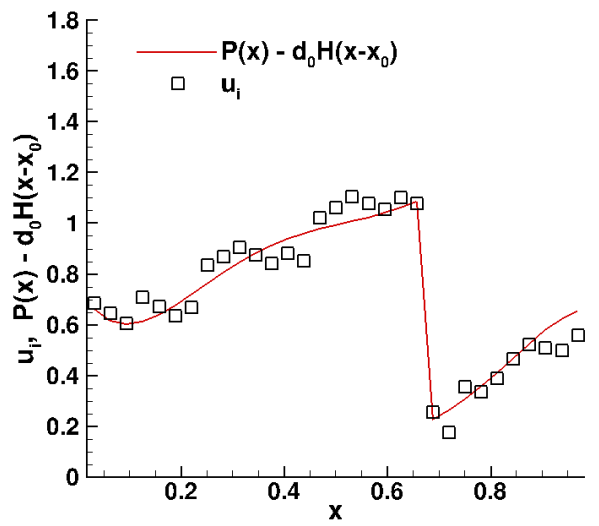

(a) Polynomial fit, 5th-order

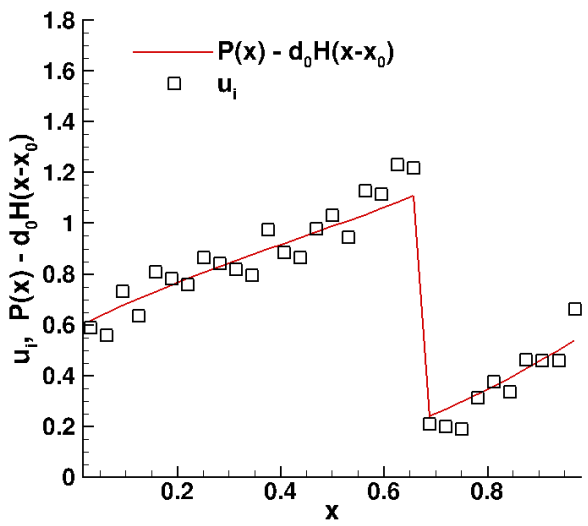

(b) Polynomial fit, 3rd-order

Figure 21. Walsh function representation of a noisy distribution with a discontinuity.

\section{Concluding Remarks}

A matrix of simulations of hypersonic flow over blunt entry vehicles with steady and pulsing retropropulsion jets is presented. Retropropulsion in the supersonic domain is primarily designed to reduce vehicle velocity directly with thrust. Retro-propulsion in the hypersonic domain may enable significant pressure recovery through unsteady, oblique shocks while providing a buffer of reactant gases with relatively low total temperature. Improved pressure recovery, a function of Mach number squared and oblique shock angle, could potentially serve to increase aerodynamic drag in this domain. The new idea here has been to explore if the aerodynamic component of the interaction can be crafted to produce significantly larger retro-force than the thrust component requiring relatively low system mass.

Pulsing jets are studied to include an additional degree of freedom to search for resonances in an already unsteady flow domain with the objective to maximize the time-averaged drag coefficient. In this paradigm, small jets with minimal footprints of the nozzle exit on the vehicle forebody may be capable of delivering the requisite perturbations to the flow. Simulations are executed assuming inviscid, symmetric flow of a perfect gas to enable a rapid assessment of the parameter space (nozzle geometry, plenum conditions, and jet pulse frequency). Solutions are generated using FUN3D for a single trajectory point at Mach 20. The focus in all cases is the time dependent variation of the drag coefficient.

Four conical nozzle configurations and three annular nozzle configurations were tested in which axisymmetric flow is assumed. Over 30 distinct conditions were simulated to include constant jet firing, pulsed jet firing, and a range of plenum conditions. The pulsed-jet configuration can produce moderately larger values of peak drag than the constant jet configurations for conditions where short pulses are separated by larger intervals characteristic of the natural frequencies observed for the steady jet interactions. Large pressures (three to four times stagnation value) can be created near the outboard shoulder, but supersonic reverse flow of a buffer gas layer (entrained propellant gas) tends to reduce surface pressure inboard and periodically shed the oblique shock impingement off the body. Consequently, the average drag coefficient is smaller than the jet-off case in all of the configurations. The annular jets showed less efficiency in perturbing the shock layer than conical jets; however, it was observed that annular jets can produce a stable buffer layer of relatively cool gas that could be beneficial for thermal protection.

The periodic shedding of buffer gas may be disrupted by introducing multiple nozzles at off-axis locations. Simulations of a periodic placement of five nozzles at different distances from the axis have been executed under the assumption that all five nozzles are fired simultaneously. It is further assumed that there is 
symmetry across the plane that passes through the axis and the center of the nozzle and across the plane that passes through the axis and midway between adjacent nozzles. The major result of these simulations is that very thin jets at off-axis locations produce chaotic, unsteady interactions, regardless if the nozzle is overexpanded or underexpanded. Large swaths of high pressure can be observed sweeping across the surface due to these interactions, but the net average drag coefficient is still less than the jet-off condition. Consequently, none of the tested configurations comes close to the target goal of doubling the drag coefficient averaged over time.

The challenges of simulating multiple, interacting shocks in this application has prompted a critical reexamination of the effects of shock capturing algorithms implicit in this problem. It is postulated that the ability of Walsh functions to simultaneously identify and quantify discontinuous jumps in a domain and produce high-order polynomial fits to that same data may be exploited to better track and resolve discontinuities in a hypersonic flow simulation. Some very preliminary examples of such an algorithm are described with a longer term goal of testing it on a problem of this complexity.

\section{Acknowledgements}

This work was sponsored by the Game Changing Development Program under the Entry Systems Modeling Project and by Revolutionary Computational Aerosciences under the Transformational Tools and Technologies project.

\section{References}

${ }^{1}$ Edquist, K.; Korzun, A.; Studak, J.; Dyakonov, A.; Shidner, J.; Kipp, D.; Tigges, M.; Prakash, R.; Trumble, K.; and Dupzyk, I.: Development of Supersonic Retro-Propulsion for Future Mars Entry, Descent, and Landing Systems. AIAA Paper 2010-5046, Jun. 2010.

${ }^{2}$ Berry, S. A.; Rhode, M. N.; and Edquist, K.: Supersonic Retropropulsion Validation Experiment in the NASA Langley Unitary Plan Wind Tunnel. J. Spacecraft and Rockets, Vol. 51, 2014, pp. 664-679.

${ }^{3}$ Berry, S. A.; Rhode, M. N.; and Edquist, K.: Supersonic Retropropulsion Experimental Results from NASA Ames 9x7 Foot Supersonic Wind Tunnel. J. Spacecraft and Rockets, Vol. 51, 2014, pp. 724-734.

${ }^{4}$ Trumble, K. A.; Schauerhamer, D. G.; Kleb, W. L.; Carlson, J.-R.; and Edquist, K. T.: Analysis of Navier-Stokes Codes Applied to Supersonic Retro-Propulsion Wind Tunnel Test. IEEE Aerospace Conference Proceedings, Apr. 2011.

${ }^{5}$ Korzun, A. M.: Aerodynamic and Performance Characterization of Supersonic retropropulsion for Application to Planetary Entry and Descent. Ph.D. Thesis, Georgia Institute of Technology, Atlanta, Georgia, May 2012.

${ }^{6}$ Bakhtian, N.; and Aftosmis, M.: Analysis of Inviscid Simulations for the Study of Supersonic Retropropulsion. AIAA Paper 2011-3194, AIAA, June 2011.

${ }^{7}$ Bakhtian, N.; and Aftosmis, M.: Maximum Attainable Drag Limits for Atmospheric Entry via Supersonic Retropropulsion. 8th International Planetary Probe Workshop, June 2011.

${ }^{8}$ Anderson, W. K.; and Bonhaus, D. L.: An Implicit Upwind Algorithm for Computing Turbulent Flows on Unstructured Grids. Comp. and Fluids, Vol. 23, no. 1, Jan. 1994, pp. 1-21.

${ }^{9}$ Biedron, R. T.; Carlson, J.-R.; Derlaga, J. M.; Gnoffo, P. A.; Hammond, D. P.; Jones, W. T.; Kleb, B.; Lee-Rausch, E. M.; Nielsen, E. J.; Park, M. A.; Rumsey, C. L.; Thomas, J. L.; and Wood, W. A.: FUN3D Manual: 12.7. NASA TM 2015-218761, NASA, May 2015.

${ }^{10}$ Walatka, P. P.; Buning, P. G.; Pierce, L.; and Elson, P.: PLOT3D User's Manual. NASA TM 101067, Mar. 1990.

${ }^{11}$ Marcum, D. L.: Advancing-Front/Local-Reconnection (AFLR) Unstructured Grid Generation. Computational Fluid Dynamics Review, World Scientific-Singapore, 1998.

${ }^{12}$ Ames Research Staff: Equations, Tables, and Charts for Compressible Flow. NACA TR 1135, 1953.

${ }^{13}$ Roe, P. L.: Discrete Models for the Numerical Analysis of Time-Dependent Multidimensional Gas Dynamics. J. Comput. Phys., Vol. 63, 1986, pp. 458-476.

${ }^{14}$ van Albada, G. D.; van Leer, B.; and Jr., W. W. R.: A comparative Study of Computational Methods in Cosmic Gas Dynamics. Astronomy and Physics, Vol. 108, no. 1, 1982, pp. 76-84.

${ }^{15}$ Huebner, L. D.; and Utreja, L. R.: Mach 10 Bow-Shock Behavior of a Forward-Facing Nose Cavity. J. Spacecraft and Rockets, Vol. 30, no. 3, May 1993, pp. 291-297.

${ }^{16}$ Stalder, J. R.; and Inouye, M.: A Method of Reducing Heat Transfer to Blunt Bodies by Air Injection. NACA RM A56B27a, May 1956.

${ }^{17}$ Pai, S. I.: Fluid Dynamics of Jets. D. Van Nostrand Company, 1954.

${ }^{18}$ Gnoffo, P. A.: Global Series Solutions of Nonlinear Differential Equations with Shocks Using Walsh Functions. J. Comput. Phys., Vol. 258, Feb 2014, pp. 650-688.

${ }^{19}$ Gnoffo, P. A.: A Walsh Function Users' Manual. NASA TM 218536, NASA, Oct. 2014.

${ }^{20}$ Gnoffo, P. A.: Unsteady Solutions of Non-Linear Differential Equations Using Walsh Function Series. AIAA Paper 2015-2756, NASA, June 2015.

${ }^{21}$ Salas, M. D.: A Shock-Fitting Primer. CRC Press, Taylor \& Francis Group, LLC, 2010. 